\title{
The endoplasmic reticulum in perisynaptic astrocytic processes: shape, distribution and effect on calcium activity
}

\author{
Audrey Denizot ${ }^{1 *}$, María Fernanda Veloz Castillo ${ }^{2}$, Pavel Puchenkov ${ }^{3}$, \\ Corrado Calì ${ }^{4,5}$, Erik De Schutter ${ }^{1}$
}

\footnotetext{
${ }^{1}$ Okinawa Institute of Science and Technology, Computational Neuroscience Unit, Onna-Son, Japan

${ }^{2}$ Biological and Environmental Science and Engineering Division, King Abdullah University of Science and Technology, Thuwal, Saudi Arabia

${ }^{3}$ Okinawa Institute of Science and Technology, Scientific Computing and Data Analysis section, Research Support Division, Onna-Son, Japan

${ }^{4}$ Department of Neuroscience, University of Torino, Italy

${ }^{5}$ Neuroscience Institute Cavalieri Ottolenghi, Orbassano, Italy
}

*To whom correspondence should be addressed; E-mail: audrey.denizot3@oist.jp.

Neurotransmission triggers $\mathrm{Ca}^{2+}$ signals in perisynaptic astrocytic processes (PAPs). As most PAPs are below the diffraction limit, the presence of $\mathrm{Ca}^{2+}$ stores in PAPs, notably the endoplasmic reticulum (ER), is unclear. Here, we create 46 three dimensional meshes of hippocampal tripartite synapses reconstructed from electron microscopy. We find that $75 \%$ of PAPs contain some $\mathrm{ER}$, as close as $72 \mathrm{~nm}$ to the synapse, and quantify its geometrical properties. To discern the effect of ER shape and distribution on $\mathrm{Ca}^{2+}$ activity, we implemented an algorithm that automatically redistributes the ER within the reconstructed PAP meshes, with constant ER and PAP shape. Reaction-diffusion simulations in those meshes reveal that $\mathrm{Ca}^{2+}$ signals in PAPs are shaped by a complex interplay between the clustering of $\mathrm{Ca}^{2+}$ channels, $\mathrm{Ca}^{2+}$ buffering, ER shape and distribution. This study, by detecting ER in PAPs and linking its spatial properties to $\mathrm{Ca}^{2+}$ activity, sheds new light on mechanisms regulating signal transmission at tripartite synapses. 


\section{Introduction}

Astrocytes are glial cells of the central nervous system that are essential to numerous brain functions (1). Notably, astrocytes are key modulators of neurotransmission at so-called tripartite synapses $(2,3)$. A single astrocyte in the CA1 region of the mouse hippocampus is in contact with hundreds of thousands of synapses simultaneously, at perisynaptic astrocytic processes (PAPs) (4). Around $75 \%$ of cortical and $65 \%$ of hippocampal synapses are contacted by an astrocytic process $(5,6)$. This close contact between astrocytes and neurons allows astrocytes to control various synaptic functions, from glutamate uptake $(7)$ and spillover $(8,9)$, to synapse homeostasis (10), stability (11), synaptogenesis (12) and neurotransmission (3, 13). Those synaptic functions are associated with specific local molecular expression in PAPs $(14,15)$, which changes upon fear conditioning (14). Importantly, the alteration of the proximity of PAPs to hippocampal synapses of the CA1 region in vivo affects neuronal activity and cognitive performance (9). Conversely, neuronal activity has been shown to induce the remodeling of synaptic coverage by PAPs in various brain regions, in vivo and in acute slices $(6,8,11,16-20)$. Together, those results illustrate that PAPs are preferential sites of neuron-astrocyte communication. The recent emergence of super-resolution techniques has provided key insights into the properties and functions of PAPs $(21,22)$. Yet, our understanding of PAP physiology and function in live tissue is hindered by the nanoscopic size of most PAPs $(23,24)$.

$\mathrm{Ca}^{2+}$ signals are commonly interpreted as a measure of astrocyte activity, notably in response to neurotransmitter release at synapses $(23,25,26)$. The recent advances in $\mathrm{Ca}^{2+}$ imaging approaches has allowed to improve the spatio-temporal resolution of $\mathrm{Ca}^{2+}$ signals in astrocytes $(26,27)$. Strikingly, it revealed that astrocytes in acute slices and in vivo exhibit spatiallyrestricted $\mathrm{Ca}^{2+}$ signals, also referred to as hotspots or microdomains, stable over time and which activity varies under physiological conditions such as locomotion or sensory stimulation (28-40). Growing evidence supports that PAPs are preferential sites displaying spatiallyrestricted $\mathrm{Ca}^{2+}$ microdomains in response to neurotransmission $(28,38,39,41,42)$. As a single astrocyte can contact hundreds of thousands of synapses simultaneously (4), such spatiallyrestricted $\mathrm{Ca}^{2+}$ microdomains might enable the astrocyte to finely tune synaptic transmission at the single synapse level.

mGluR activation on the astrocytic membrane following neurotransmission at glutamatergic synapses results in $\mathrm{Ca}^{2+}$ transients mediated by $G_{q}$ proteins and $\mathrm{Ca}^{2+}$ stores such as the endoplasmic reticulum (ER) (27). Most astrocytic $\mathrm{Ca}^{2+}$ signals are mediated by the Inositol 3Phosphate $\left(\mathrm{IP}_{3}\right)$ receptors on the membrane of the endoplasmic reticulum (ER) (43). Because of their nanoscopic size, the $\mathrm{Ca}^{2+}$ pathways involved in microdomain $\mathrm{Ca}^{2+}$ signals in PAPs are still unclear. Notably, the presence of ER in PAPs and its involvement in microdomain $\mathrm{Ca}^{2+}$ signals at synapses is highly debated. During the last decade, PAPs have indeed been regarded as devoid of ER, with a minimum distance between the synapse and the closest astrocytic ER > $0.5 \mu \mathrm{m}(23,44)$. In contrast, inhibiting ER-mediated $\mathrm{Ca}^{2+}$ signaling in fine processes results in a 
decreased number of $\mathrm{Ca}^{2+}$ domains $(30)$ and a decreased $\mathrm{Ca}^{2+}$ peak frequency $(30,37,42)$. Furthermore, some astrocytic ER has been detected near synapses in other EM studies $(24,45,46)$. Yet, the geometrical properties of the ER in PAPs and its distribution remain poorly characterized, but could have a strong impact on neuron-astrocyte communication at tripartite synapses.

Here, we use a $220 \mu \mathrm{m}^{3}$ hippocampal astrocytic volume from the CA1 stratum radiatum region (6 $\mathrm{nm}$ voxel resolution) (47), reconstructed from electron microscopy (EM), to create 46 three dimensional meshes of tripartite synapses. Strikingly, we find that $75 \%$ of PAPs contain some ER, which can be as close as $72 \mathrm{~nm}$ to the post-synaptic density (PSD). Analysis of the geometrical features of those meshes reveal the vast diversity of ER shapes and distributions within PAPs from a single cell. We then used a detailed stochastic reaction-diffusion model of $\mathrm{Ca}^{2+}$ signals in PAPs to investigate the mechanistic link between the spatial features of the ER measured in the 3D meshes and the spatio-temporal properties of $\mathrm{Ca}^{2+}$ microdomain activity in PAPs. To be able to decipher the effect of ER distribution within the PAP independently from the effect of its shape, we developed an algorithm that automatically creates realistic 3D tetrahedral PAP meshes with various ER distributions from the realistic meshes reconstructed from EM. In silico experiments in those meshes reveal that the spatio-temporal properties of $\mathrm{Ca}^{2+}$ signals in PAPs are tightly regulated by a complex interplay between the clustering of $\mathrm{Ca}^{2+}$ channels, the ratio between ER surface area and PAP volume, $\mathrm{Ca}^{2+}$ buffering and ER spatial distribution. Together, this study provides new insights into the geometrical properties of hippocampal tripartite synapses and predicts mechanistic links between those features and $\mathrm{Ca}^{2+}$ microdomain activity at tripartite synapses.

\section{Results}

\section{Quantification of the main geometrical properties of hippocampal tripar- tite synapses}

To characterize the presence, shape and distribution of the endoplasmic reticulum (ER) in perisynaptic astrocytic processes (PAPs), we used a $220 \mu \mathrm{m}^{3}(7.07 \mu \mathrm{m} \times 6.75 \mu \mathrm{m} \times 4.75 \mu \mathrm{m})$ hippocampal astrocytic volume from the CA1 stratum radiatum region reconstructed from a perfectly isotropic EM stack (6 nm voxel resolution) (47). Elements from the neuropil, i.e. boutons, dendritic spines and post-synaptic densities (PSDs), were also reconstructed. Following the workflow presented in Fig. 1A, 44 excitatory and 2 inhibitory tripartite synapse meshes were created, containing all elements belonging to the astrocyte and to the neuropil within a $3.375 \mu \mathrm{m}^{3}$ volume, corresponding to a cube of $1.5 \mu \mathrm{m}$ edge length centered at the center of mass of the PSD. Five of those tripartite synapse meshes are displayed in Fig. 1B. Among those meshes, 16 were located at the borders of the $220 \mu \mathrm{m}^{3}$ astrocytic volume. They were thus omitted from data analysis as synaptic elements in those meshes could not be fully reconstructed. The volume, surface area and surface-volume ratio (SVR) of each synaptic element, 


\section{$\mathbf{A}$}
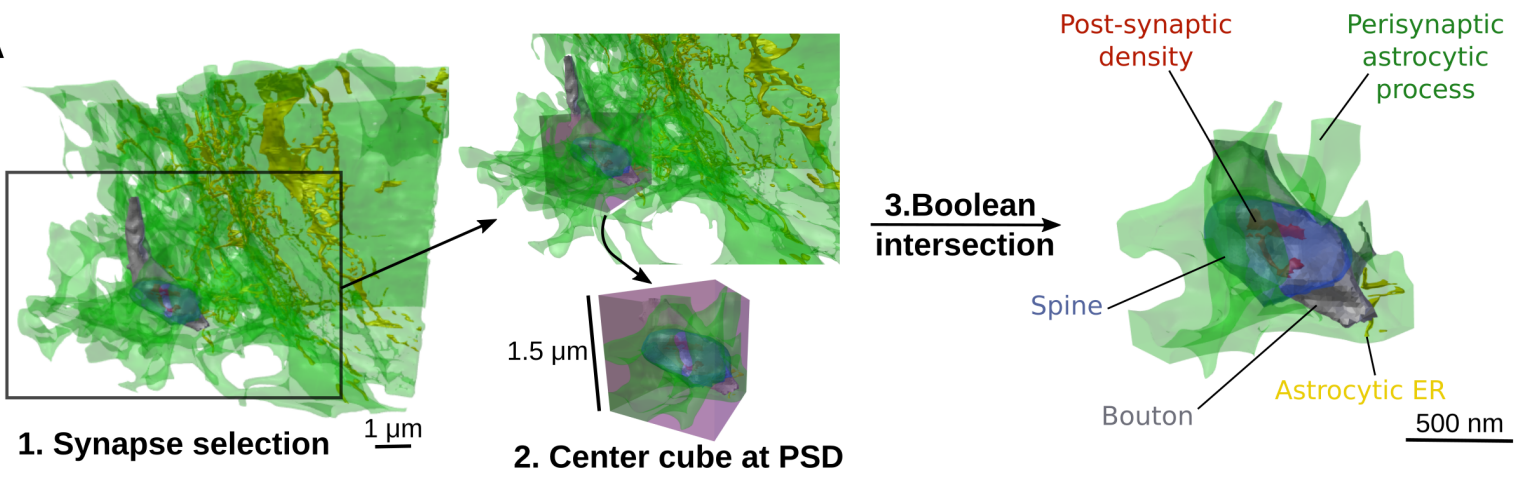

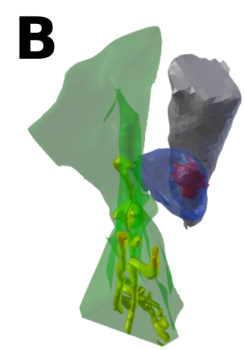

d1s1a25b2

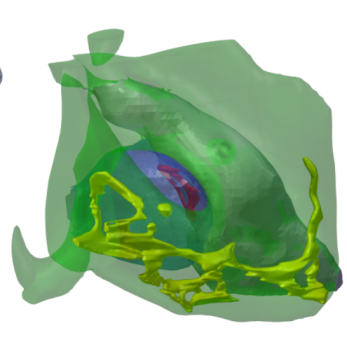

d1s15a32b1

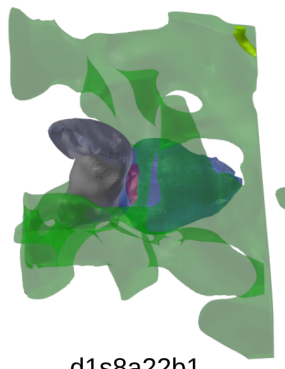

d1s8a22b1

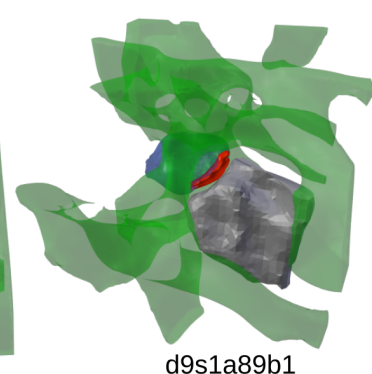

d9s1a89b1

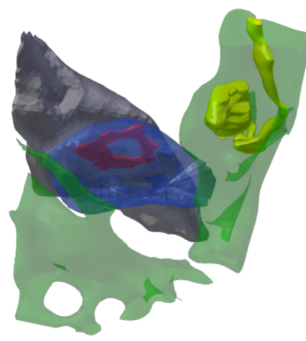

d1s3a107b1

$500 \mathrm{~nm}$

Fig 1: Reconstruction of $\mathbf{4 6}$ tripartite synapse meshes from electron microscopy. (A) Schematic presenting our tripartite synapse mesh creation workflow, here performed on synapse d10s1a2b1. 1. Synapses in contact with the $220 \mu \mathrm{m}^{3}$ astrocytic volume were selected one by one. 2. A cube of $1.5 \mu \mathrm{m}$ edge length $\left(3.375 \mu \mathrm{m}^{3}\right)$ was created and centered at the center of mass of the post-synaptic density (PSD, red). 3. Boolean intersection between the neuronal and astrocytic objects and the cube resulted in the isolation of the elements of the tripartite synapse mesh: the perisynaptic astrocytic process (PAP, green), the astrocytic endoplasmic reticulum (ER, yellow), the bouton (grey) and the spine (blue). This workflow resulted in the creation of 44 excitatory and 2 inhibitory tripartite synapse meshes. (B) Images of five of the 3D tripartite synapse meshes created, d1s1a25b2, d1s15a32b1, d1s8a22b1, d9s1a89b1, d1s3a107b1, revealing their diverse geometrical properties. 
i.e the PAP, astrocytic ER, spine and bouton, of the remaining 28 fully reconstructed excitatory tripartite synapses are presented in Fig. 2C-E and Supplemental Table S1. The minimum distance between each vertex on the membrane of the PAP and the center of mass of the PSD was measured in each of the 28 meshes (Fig. 2B), providing a quantification of the distribution of the astrocyte around the synapse. Our results highlight the diverse distances between PSDs and PAPs belonging to a single cell. In accordance with previous studies $(6,44,48)$, PAP membrane vertices could be as close as $5 \mathrm{~nm}$ to the PSD, with an average distance between the PSD and the closest PAP vertex of $65 \mathrm{~nm}$. Importantly, we found that PM-PSD distance is the shortest, i.e PAPs are the closest to the synapse, when bouton surface area is low (Fig. 2F, p=0.013). PAP-PSD distance was not correlated to PAP (Fig. 2G, $\mathrm{p}=0.14$ ) or spine (Fig. 2H, $\mathrm{p}=0.24$ ) surface area.

\section{Presence and geometrical properties of the endoplasmic reticulum in perisy- naptic astrocytic processes}

Because of the small size of most PAPs, the $\mathrm{Ca}^{2+}$ pathways that regulate astrocytic $\mathrm{Ca}^{2+}$ microdomain activity at tripartite synapses remain to be uncovered. Notably, the presence of ER in PAPs is controversial $(24,44-46)$. We have thus analyzed the presence and shape of the ER in the PAPs from the 28 fully reconstructed excitatory tripartite synapse meshes presented in Fig. 2.

75\% of PAPs contained some ER (Fig. 3C), which challenges the widespread belief that tripartite synapses are devoid of astrocytic ER. ER surface area, volume and SVR were measured in ER-containing PAPs and highlight that ER shape is highly variable between PAPs from the same cell (Fig. 3B). Note that there was no significant difference between bouton, spine and PAP surface area, volume and SVR between synapses with vs without astrocytic ER (Supplemental Fig. S1). We further characterized the vicinity of the astrocytic ER to the synapse. To do so, we measured the distance between each vertex on the ER membrane to the center of mass of the PSD $(n=21)$. We found that ER-PSD distance varies drastically from synapse to synapse (Fig. 3E) and can be as little as $72 \mathrm{~nm}$, far below the $>0.5 \mu \mathrm{m}$ ER-PSD distance reported previously $(23,44)$. The closest ER vertex was on average $432 \mathrm{~nm}$ away from the center of mass of the PSD. Interestingly, the larger the surface area of the ER, the closer it was to the PSD (Fig. 3F, p=0.013). Astrocytic ER was closer to the PSD in PAPs with higher surface area (Fig. 3G, p=0.024). The minimum ER-PSD distance was not correlated to spine (Fig. 3H, $\mathrm{p}=0.54$ ) or bouton (Fig. 3I, p=0.29) surface area. Overall, our results highlight that most PAPs contain some ER and that its shape is highly variable, which could have strong implications on ER-dependent $\mathrm{Ca}^{2+}$ signaling in PAPs resulting from synaptic transmission. 

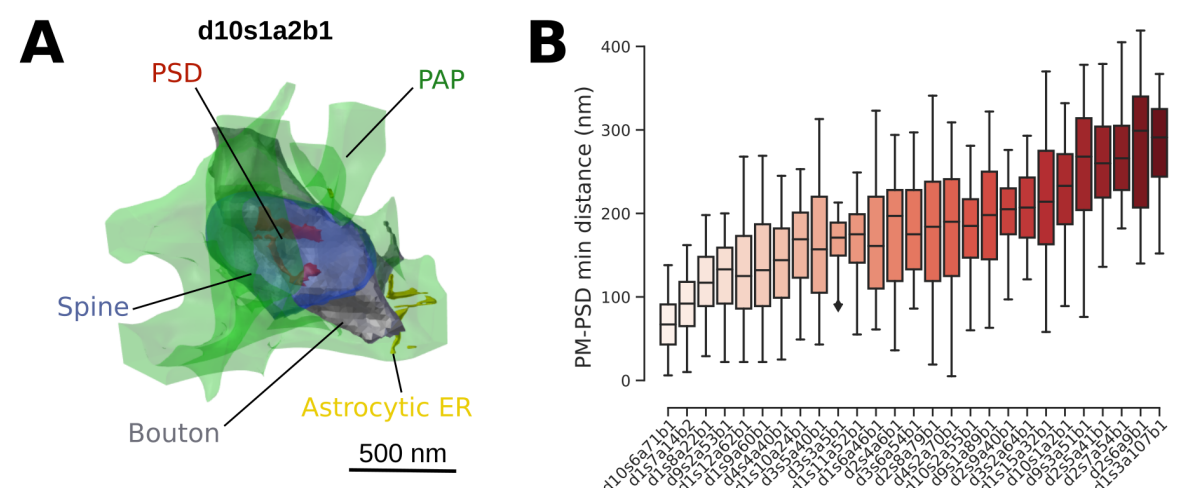

$500 \mathrm{~nm}$
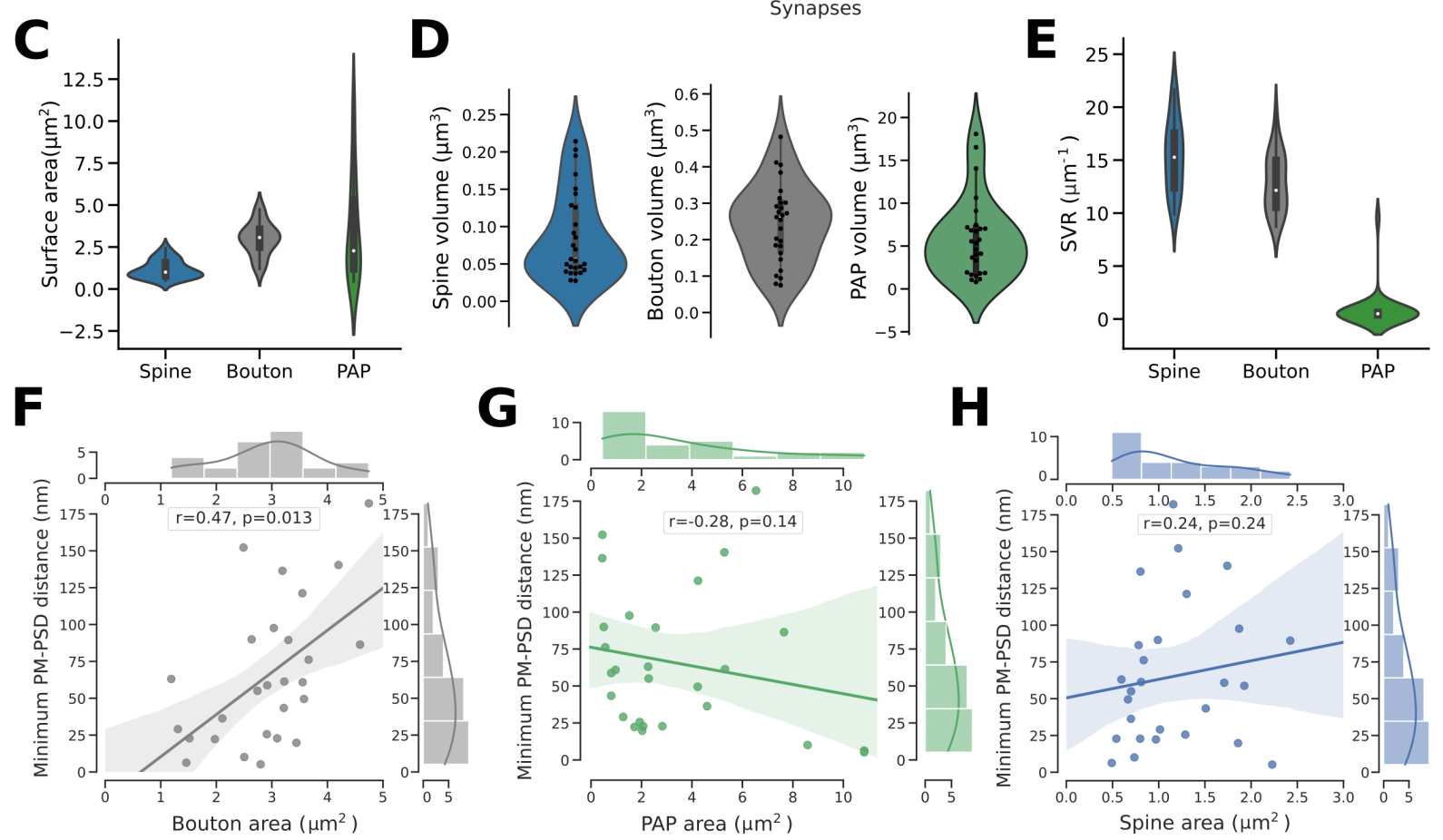

Fig 2: Characterization of the geometrical properties of hippocampal tripartite synapses. (A) Image of a tripartite synapse mesh, d10s1a2b1, containing a bouton (grey), spine (blue), post-synaptic density (PSD, red), perisynaptic astrocytic process (PAP, green) and the astrocytic endoplasmic reticulum (ER, yellow). (B) Left: Boxplots presenting the distribution of the minimum distance between each vertex on the PAP membrane and the center of mass of the PSD, measured in the 28 excitatory tripartite synapse meshes fully reconstructed in this study. Right: Distribution of the minimum distance between the PAP and the PSD ( $n=28)$. (C-E) Violin plots presenting the distribution of spine, bouton and PAP surface area (C), volume (D) and surface-volume ratio (E). (F-H) Scatterplots presenting the variation of the minimum PAP-PSD distance as a function of bouton surface area (left), PAP surface area (middle) and spine surface area (right). Plots are presented with univariate kernel density estimation curves and a linear regression fit. Spearman correlation coefficients, $r$, and $p$-values, $p$, are displayed onto each regression plot, $\mathrm{n}=28$. 


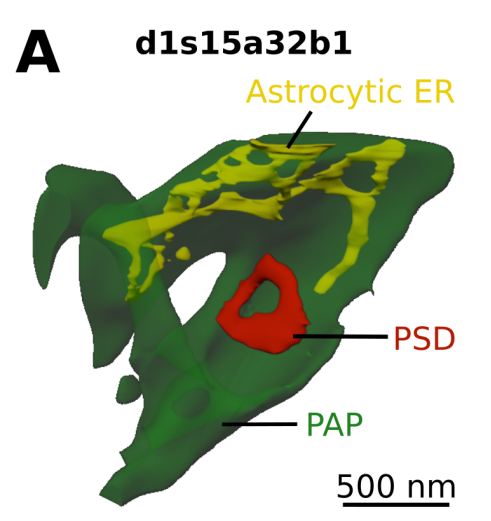

$\mathbf{E}$
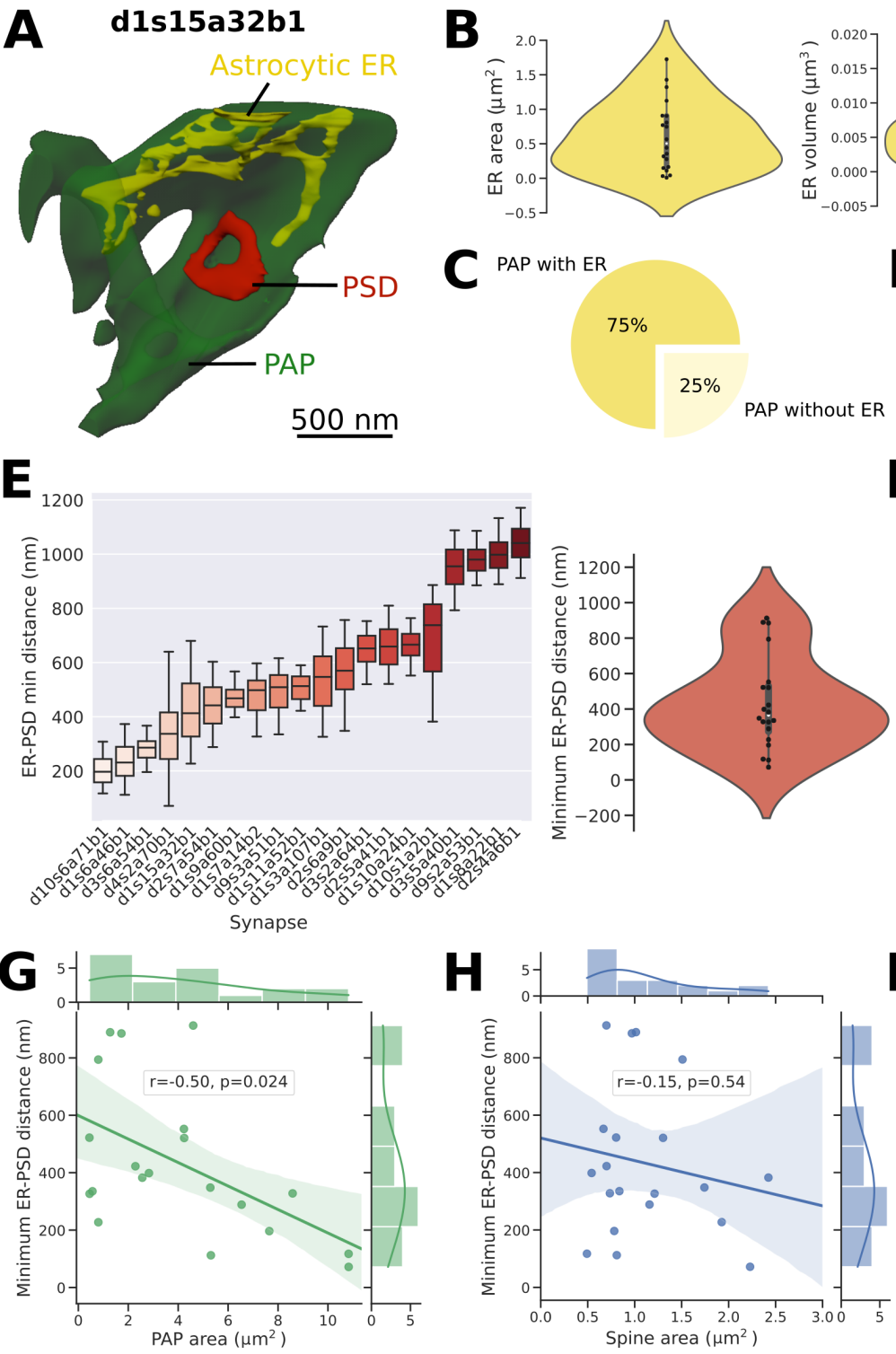
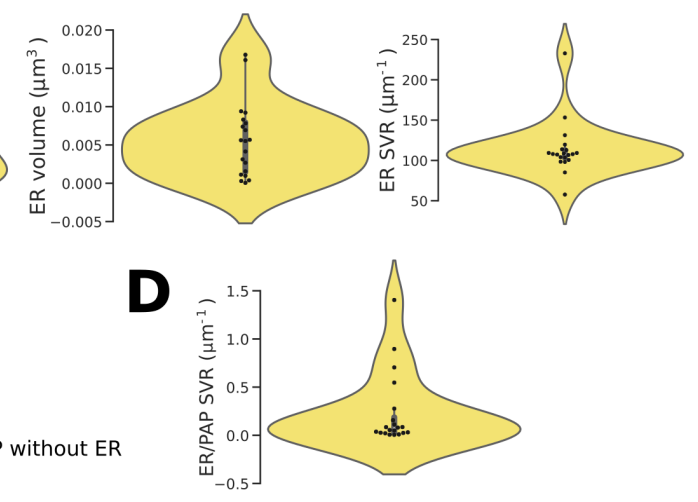

$\mathbf{F}$
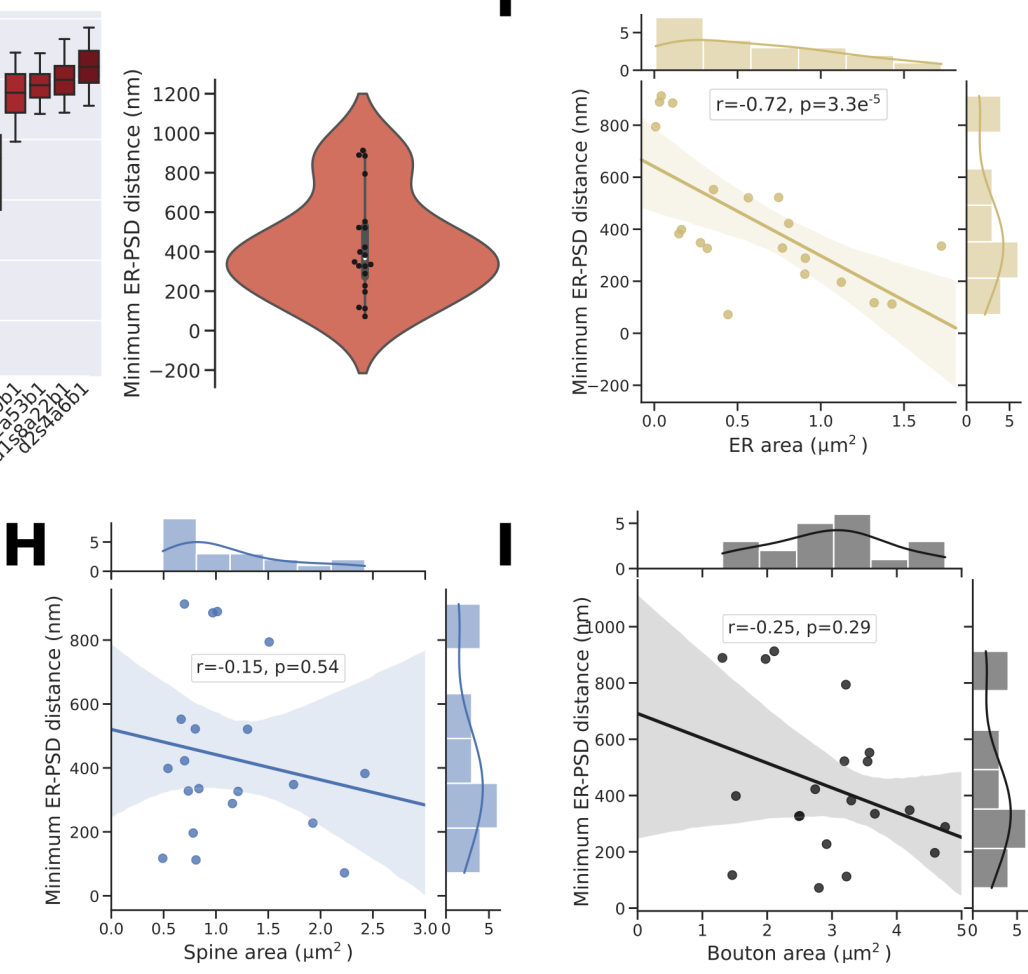

Fig 3: Presence and geometrical properties of the endoplasmic reticulum in perisynaptic astrocytic processes. (A) Image of the $\mathrm{d} 1 \mathrm{~s} 15 \mathrm{a} 32 \mathrm{~b} 1 \mathrm{PSD}$ (red) and the neighboring PAP (green), that contains ER (yellow). (B) Violin plots representing the distribution of ER surface area (left), volume (middle) and surface volume ratio (right) within PAPs, $n=21$. (C) Among the 28 fully reconstructed PAP meshes extracted, $75 \%$ contained some ER. (D) Distribution of the ratio between the ER surface area and PAP volume $(n=21)$. (E) Quantitative analysis of the distance between the astrocytic ER and the neighboring PSD, $n=21$. (Left) Boxplots presenting the distribution of the distance of ER membrane vertices to the center of mass of the PSD in each PAP. (Right) Distribution of the minimum ER-PSD distance in PAPs, $n=21$. The lowest ER-PSD distance measured was 70nm (synapse d4s2a70b1). 
Fig 3: (F-I) Scatterplots presenting the variation of the minimum ER-PSD distance as a function of ER surface area (F), PAP surface area $(\mathrm{G})$, spine surface area $(\mathrm{H})$ and bouton surface area (I), $\mathrm{n}=21$. Plots are presented with univariate kernel density estimation curves and a linear regression fit. Spearman correlation coefficient, $r$, and $p$-value, $p$, are displayed onto each regression plot.

\section{Reaction-diffusion simulations reveal different spatio-temporal properties of $\mathrm{Ca}^{2+}$ signals in PAPs of the same cell}

PAPs are characterized by highly diverse sizes and shapes of the ER (Fig. 3), which could have strong implications on ER-mediated $\mathrm{Ca}^{2+}$ signals in PAPs. Because of their nanoscale, measuring $\mathrm{Ca}^{2+}$ activity and deciphering the involvement of ER-mediated signals in individual PAPs in live tissue is extremely challenging (26). A better understanding of the mechanistic link between the geometrical properties of the ER and the spatio-temporal properties of $\mathrm{Ca}^{2+}$ microdomain signals in PAPs is crucial, yet hard to test experimentally. Here, we use the PAP meshes presented in Fig. 3 together with a spatial stochastic model of $\mathrm{Ca}^{2+}$ signaling adapted from the model of Denizot and collaborators (49) to investigate the mechanistic link between ER shape and $\mathrm{Ca}^{2+}$ microdomain activity in PAPs. $\mathrm{Ca}^{2+}$ influx in the PAP cytosol in the model is mediated by Inositol 3-Phosphate $\left(\mathrm{IP}_{3}\right)$ receptors on the membrane of the ER and by $\mathrm{Ca}^{2+}$ channels at the plasma membrane, $C h_{\mathrm{PM}}$. The reactions modeled are presented in Fig. $4 \mathrm{~A}$ and in the Methods section. Neuronal activity was simulated at $\mathrm{t}=1 \mathrm{~s}$ by infusing $50 \mathrm{IP}_{3}$ molecules at the PM of the PAP. The implementation of this model with STEPS software (50) allows to perform simulations in tetrahedral meshes in 3 spatial dimensions, such as the ones created in this study. Representative Ca-GCaMP traces in a cylindrical mesh, corresponding to the concentration of $\mathrm{Ca}^{2+}$ bound to $\mathrm{Ca}^{2+}$ indicators added to the cytosol of the model, display similar spatio-temporal characteristics to $\mathrm{Ca}^{2+}$ signals measured in organotypic hippocampal astrocytic cultures (51) (Fig. 4A, right).

We performed simulations in six PAP meshes reconstructed from electron microscopy, characterized by various geometrical properties of the ER: d1s3a107b1, d1s8a22b1, d1s10a24b1, d2s6a9b1, d9s4a34b1 and d10s1a2b1 (Fig. 4B, Table 1). To do so, meshes were pre-processed to allow their use in reaction-diffusion simulations. The pre-processing workflow is described in Fig. 4C and in the Methods section, and produced 3D tetrahedral meshes from the 2D triangular meshes reconstructed from EM. Screenshots of simulations performed in two realistic tetrahedral PAP meshes are presented in Fig. 4B. Ca-GCaMP and free $\mathrm{Ca}^{2+}$ signals, in simulations with and without $\mathrm{Ca}^{2+}$ indicators in the cytosol, respectively, were measured in d1s3a107b1, d1s8a22b1, d1s10a24b1, d2s6a9b1, d9s4a34b1 and d10s1a2b1 PAP meshes. Representative traces are displayed in Fig. 4E. Signals varied greatly depending on the mesh (Fig. 4F). Note that, in accordance with previous studies $(49,52), \mathrm{Ca}-\mathrm{GCaMP}$ and free $\mathrm{Ca}^{2+}$ signals displayed different spatio-temporal properties (Fig. 4F). Those results suggest that the diverse geometrical 
Table 1: Characteristics of the 3D PAP meshes used in the reaction-diffusion simulations. $\mathrm{V}_{\text {cyt }}$ is the cytosolic volume, $\mathrm{S}_{\mathrm{PM}}$ is the plasma membrane surface area, $\mathrm{S}_{\mathrm{ER}}$ is the ER surface area, $\mathrm{SVR}_{\mathrm{ER} / \mathrm{PAP}}$ is the ratio between the ER surface area and the cytosolic volume. $E R_{\mathrm{c}}$ is the number of ER vertices at ER-PM contact sites, i.e $\leq 20 \mathrm{~nm}$ from the closest PM vertex. $\mathrm{d} 1 \mathrm{~s} 15 \mathrm{a} 32 \mathrm{~b} 1_{\mathrm{f} 0}, \mathrm{~d} 1 \mathrm{~s} 15 \mathrm{a} 32 \mathrm{~b} 1_{\mathrm{f} 21}, \mathrm{~d} 1 \mathrm{~s} 15 \mathrm{a} 32 \mathrm{~b} 1_{\mathrm{f} 64}$ and $\mathrm{d} 1 \mathrm{~s} 15 \mathrm{a} 32 \mathrm{~b} 1_{\mathrm{f} 250}$ refer to meshes from frames 0, 21, 64 and 250 of the d1s15a32b1 PAP mesh presented in Fig. 8-9.

\begin{tabular}{|llllll|}
\hline Geom & $\mathrm{V}_{\text {cyt }}\left(\mu m^{3}\right)$ & $\mathrm{S}_{\mathrm{PM}}\left(\mu m^{2}\right)$ & $\mathrm{S}_{\mathrm{ER}}\left(\mu m^{2}\right)$ & $\mathrm{SVR}_{\mathrm{ER} / \mathrm{PAP}}\left(\mu m^{-1}\right)$ & $E R_{\mathrm{c}}$ \\
\hline$d 1 s 3 a 107 b 1$ & 0.112 & 2.00 & 0.315 & 2.81 & 183 \\
\hline$d 1 s 8 a 22 b 1$ & 0.397 & 8.60 & 0.031 & 0.078 & 31 \\
\hline$d 1 s 10 a 24 b 1$ & 0.331 & 5.80 & 0.344 & 1.04 & 0 \\
\hline$d 2 s 6 a 9 b 1$ & 0.505 & 10.0 & 0.273 & 0.54 & 20958 \\
\hline$d 9 s 4 a 34 b 1$ & 0.410 & 7.05 & 0.807 & 1.97 & 3771 \\
\hline$d 10 s 1 a 2 b 1$ & 0.531 & 10.0 & 0.136 & 0.26 & 295 \\
\hline $\mathrm{d} 1 \mathrm{~s} 15 \mathrm{a} 32 \mathrm{~b} 1_{\mathrm{f} 0}$ & 0.426 & 6.91 & 0.85 & 2.00 & 2337 \\
\hline $\mathrm{d} 1 \mathrm{~s} 15 \mathrm{a} 32 \mathrm{~b} 1_{\mathrm{f} 21}$ & 0.426 & 6.91 & 0.85 & 2.00 & 1683 \\
\hline $\mathrm{d} 1 \mathrm{~s} 15 \mathrm{a} 32 \mathrm{~b} 1_{\mathrm{f} 64}$ & 0.426 & 6.91 & 0.85 & 2.00 & 0 \\
\hline $\mathrm{d} 1 \mathrm{~s} 15 \mathrm{a} 32 \mathrm{~b} 1_{\mathrm{f} 250}$ & 0.426 & 6.91 & 0.85 & 2.00 & 0 \\
\hline $\mathrm{PAP} 1_{\mathrm{v}}$ & 0.434 & 3.55 & 0.088 & 0.21 & 125 \\
\hline PAP $1_{\mathrm{w}}$ & 0.432 & 3.55 & 0.428 & 0.99 & 0 \\
\hline PAP $_{\mathrm{x}}$ & 0.428 & 3.55 & 0.834 & 1.95 & 555 \\
\hline PAP $_{\mathrm{y}}$ & 0.423 & 3.55 & 1.27 & 3.00 & 3.88 \\
\hline PAP $_{\mathrm{z}}$ & 0.418 & 3.55 & 1.62 & & \\
\hline
\end{tabular}

features of PAPs and ER reported in this study (Fig. 2-3) strongly influence $\mathrm{Ca}^{2+}$ microdomain activity at tripartite synapses.

\section{The effect of IP3R clustering differs from PAP to PAP}

$\mathrm{IP}_{3} \mathrm{R}$ channels are not randomly distributed on the surface of the ER and form stable clusters of various sizes (53-56). Numerous computational studies performed in 2 spatial dimensions have predicted that $\mathrm{IP}_{3} \mathrm{R}$ cluster size shapes $\mathrm{Ca}^{2+}$ activity (see (57) for a review). Whether this effect still holds in 3D, notably in complex shapes such as that of the PAPs reconstructed in this study, remains to be uncovered. We thus next simulated $\mathrm{Ca}^{2+}$ signaling in the 6 realistic PAP meshes presented in Fig. 4, reconstructed from EM, d1s3a197b1, d1s8a22b1, d1s10a24b1, d2s6a9b1, d9s4a34b1 and d10s1a2b1, with various distributions of $\mathrm{IP}_{3} \mathrm{Rs}$ on the membrane of the ER.

Simulations of the model were performed with different $\mathrm{IP}_{3} \mathrm{R}$ cluster sizes $\eta$. $\mathrm{Ca}^{2+}$ channels were either randomly placed on the membrane of the PAP $(\operatorname{cocl}=0)$ or clustered onto the $\mathrm{PM}$ triangles that were the closest to the ER triangles containing an $\mathrm{IP}_{3} \mathrm{R}$ cluster $(\mathrm{cocl}=1)$, emulating co-localization of $\mathrm{Ca}^{2+}$ channels, reported in neurons and astrocytes (58). As $\mathrm{IP}_{3} \mathrm{R}$ 
A
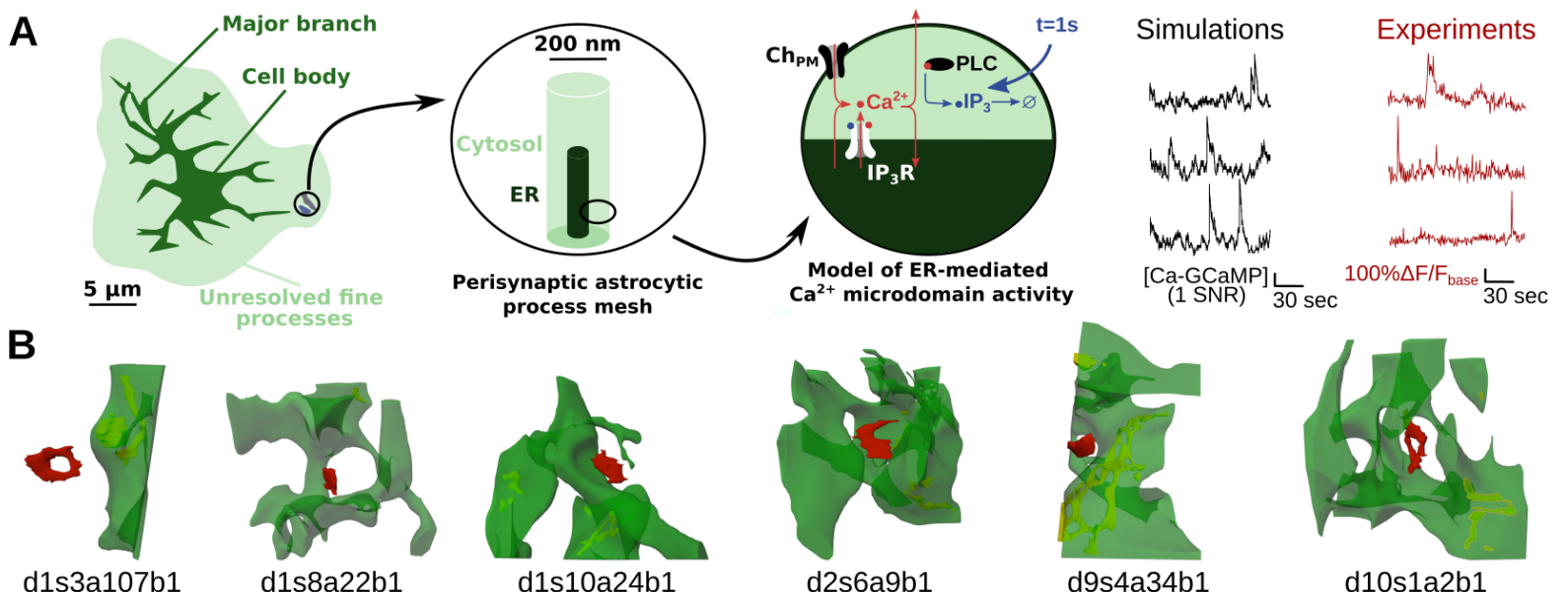
processes

Model of ER-mediated
$\mathrm{Ca}^{2+}$ microdomain activity $\underset{(1 \mathrm{SNR})}{[\mathrm{Ca}-\mathrm{GCaMP}]} \underset{30 \mathrm{sec}}{L}$
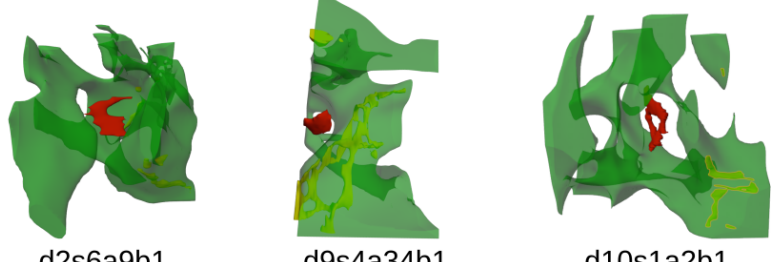

d1s10a24b1

$d 2 s 6 a 9 b 1$

d9s4a34b1

$\mathrm{d} 10 \mathrm{~s} 1 \mathrm{a} 2 \mathrm{~b} 1$
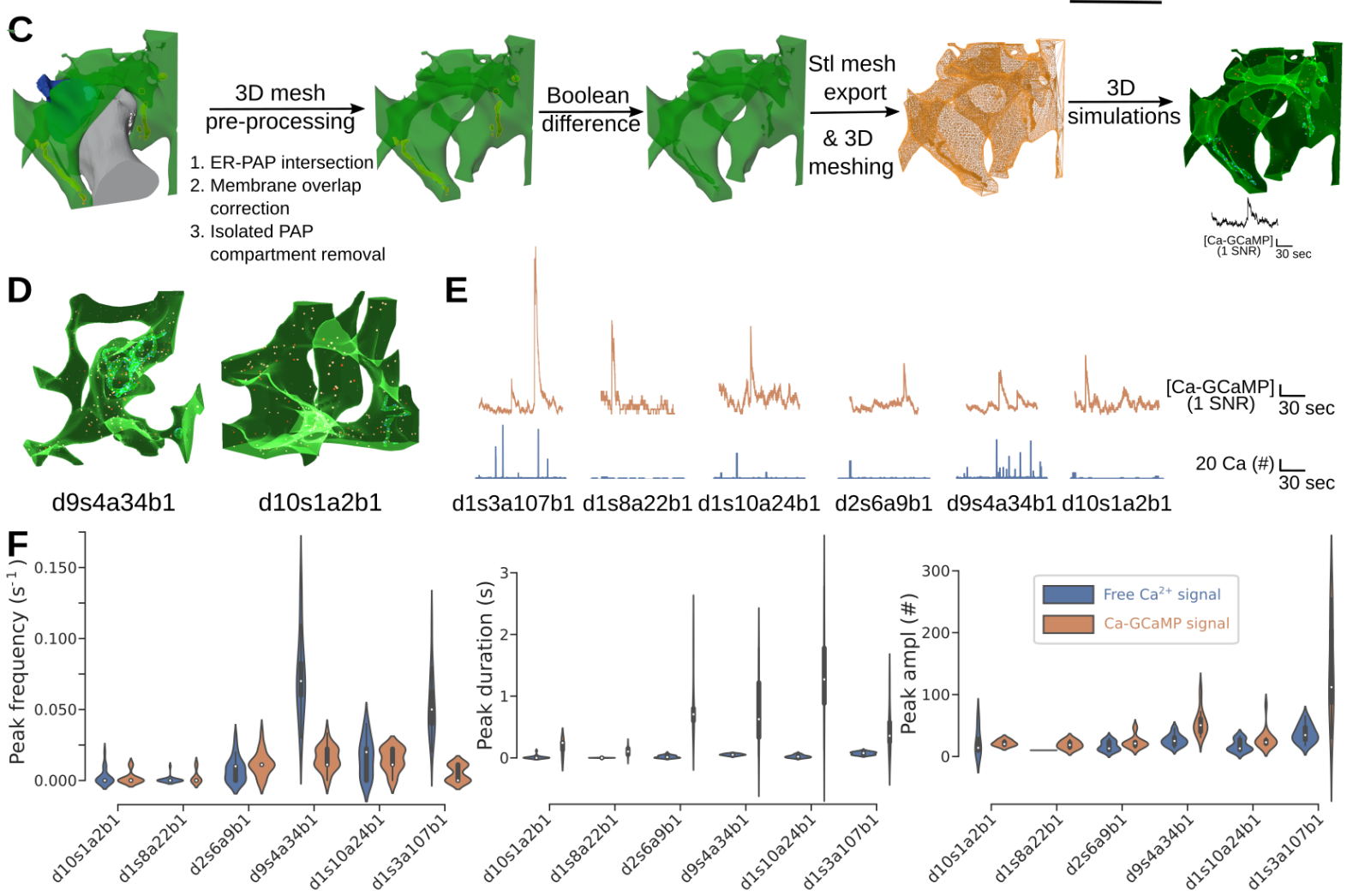

Fig 4: Reaction-diffusion simulations reveal different spatio-temporal properties of $\mathrm{Ca}^{2+}$ signals between PAPs of the same cell. (Left) Schematic representation of the model of $\mathrm{Ca}^{2+}$ signaling in PAPs used in this study. The model is stochastic, spatially-extended and simulations can be performed in 3D meshes. $\mathrm{Ca}^{2+}$ influx into the cytosol results from $\mathrm{Ca}^{2+}$ channels on the plasma membrane and from $\mathrm{IP}_{3} \mathrm{R}$ channels on the ER. At $\mathrm{t}=1 \mathrm{~s}, 50 \mathrm{IP}_{3}$ molecules were injected at the plasma membrane of the PAP, simulating neuronal activity. (Right) Representative CaGCaMP traces from simulations in a cylindrical mesh, $200 \mathrm{~nm}$ in diameter, $1 \mu \mathrm{m}$ long (left, black) and experiments (right, red) (51). 
Fig 4: (B) Images representing the 6 PAP meshes in which simulations were performed: d1s3a197b1, d1s8a22b1, d1s10a24b1, d2s6a9b1, d9s4a34b1 and d10s1a2b1. (C) Workflow to prepare the PAP meshes for 3D simulations illustrated on d2s6a9b1 mesh (see Methods section). The geometrical features of the resulting PAP meshes are presented in Table 1. (D) Screenshots of simulations in PAP meshes d9s4a34b1 and d10s1a2b1. Note that the darker and lighter greens result from 3D shading and rendering of the meshes. (E) Representative CaGCaMP (top, orange) and free $\mathrm{Ca}^{2+}$ (bottom, blue). $\mathrm{Ca}^{2+}$ traces were measured in separate simulations, where no GCaMP was added into the cytosol of the PAP. $\mathrm{IP}_{3} \mathrm{R}$ channels and $\mathrm{Ca}^{2+}$ channels at the plasma membrane, $\mathrm{Ch}_{\mathrm{PM}}$, were randomly distributed onto the ER membrane and plasma membrane, respectively. (F) Quantification of peak frequency (left), duration (middle) and amplitude (right) of free $\mathrm{Ca}^{2+}$ (left, blue, $\mathrm{n}=20$ ) and Ca-GCaMP (right, orange, $\mathrm{n}=20$ ) signals measured in silico in $3 \mathrm{D}$ meshes of the PAPs presented in panel B.

density was kept constant across simulations, $3.5 e^{-3} / \mu m^{2}(49)$, the total number of $\mathrm{IP}_{3} \mathrm{Rs}_{\text {, }}$ $N_{\text {IP3R }}$, varied depending on the mesh: $90,230,78,8,40$ and 96 in PAP meshes from synapses d1s3a107b1, d9s4a34b1, d2s6a9b1, d1s8a22b1, d10s1a2b1 and d1s10a24b1, respectively. As $\mathrm{IP}_{3} \mathrm{R}$ cluster size was a divider of $N_{\mathrm{IP} 3 \mathrm{R}}$, cluster sizes tested varied slightly depending on the mesh. The range of $\mathrm{IP}_{3} \mathrm{R}$ cluster size tested varied from $\eta=1-26$. Representative free $\mathrm{Ca}^{2+}$ traces measured in d1s3a197b1, d1s8a22b1, d1s10a24b1, d2s6a9b1, d9s4a34b1 and d10s1a2b1 PAP meshes with various $\mathrm{IP}_{3} \mathrm{R}$ cluster sizes $\eta$ are displayed in Fig. 5B. Strikingly, $\mathrm{IP}_{3} \mathrm{R}$ clustering only affected $\mathrm{Ca}^{2+}$ activity in a subset of the PAP meshes studied (Fig. 5C). Indeed, $\mathrm{Ca}^{2+}$ peak duration and amplitude increased with $\mathrm{IP}_{3} \mathrm{R}$ cluster size in PAP meshes from synapses $\mathrm{d} 1 \mathrm{~s} 3 \mathrm{a} 107 \mathrm{~b} 1$ (ANOVA, $\mathrm{p}=5.1 e^{-5}$ and $1.9 e^{-7}$ ), d2s6a9b1 (ANOVA, $\mathrm{p}=3.16 e^{-3}$ and 0.026) and $\mathrm{d} 9 \mathrm{~s} 4 \mathrm{a} 34 \mathrm{~b} 1$ (ANOVA, $\mathrm{p}=0.018$ and 0.028). This effect was associated with an increased frequency of $\mathrm{IP}_{3} \mathrm{R}$ opening with cluster size (ANOVA, $\mathrm{p}=3.4 e^{-4}, 0.007$ and 0.037 in $\mathrm{d} 1 \mathrm{~s} 3 \mathrm{a} 107 \mathrm{~b} 1$, d2s6a9b1 and d9s4a34b1, respectively). Those results highlight that different PAP and ER shapes are associated with different $\mathrm{IP}_{3} \mathrm{R}$ clustering effects.

Interestingly, increased neuronal stimulation, simulated as an increased amount of $\mathrm{IP}_{3}$ infused, $i$, in the PAP at $\mathrm{t}=1 \mathrm{~s}$, triggered clustering effects in a PAP in which no clustering effect was observed after a milder neuronal stimulation (Fig. 5D). This effect was characterized by an increase of $\mathrm{Ca}^{2+}$ peak amplitude (ANOVA, $\mathrm{p}=8.2 e^{-5}$ for $\mathrm{i}=150$ and $\mathrm{p}=4.66 e^{-7}$ for $\mathrm{i}=200$ ), frequency (ANOVA, $\mathrm{p}=0.005$ for $\mathrm{i}=150$ and $\mathrm{p}=0.006$ for $\mathrm{i}=200$ ) and duration (ANOVA, $\mathrm{p}=3.4 e^{-5}$ for $i=150$, and $p=0.026$ for $i=200$ ) with $\mathrm{IP}_{3} \mathrm{R}$ cluster size for $\mathrm{i}=150$ and 200 , while cluster size did not affect peak amplitude (ANOVA, $\mathrm{p}=0.27$ for $\mathrm{i}=50$ and $\mathrm{p}=0.08$ for $\mathrm{i}=100$ ), frequency (ANOVA, $\mathrm{p}=0.86$ for $\mathrm{i}=50$ and $\mathrm{p}=0.72$ for $\mathrm{i}=100)$ and duration $(\mathrm{p}=0.13$ for $\mathrm{i}=50$ and $\mathrm{p}=0.15$ for $\mathrm{i}=100$ ) for $\mathrm{i}=50$ and 100 . This effect was mediated by an increased $\mathrm{IP}_{3} \mathrm{R}$ opening frequency with cluster size when neuronal stimulation was larger (ANOVA, $\mathrm{p}=0.024,5.23 e^{-7}$ and $7.8 e^{-5}$, for $\mathrm{i}=100,150$ and 200, respectively), while clustering had no effect on $\mathrm{IP}_{3} \mathrm{R}$ opening frequency for milder neuronal stimulation (ANOVA, $\mathrm{p}=0.21, \mathrm{i}=50$ ). This suggests that $\mathrm{IP}_{3} \mathrm{R}$ clustering in 

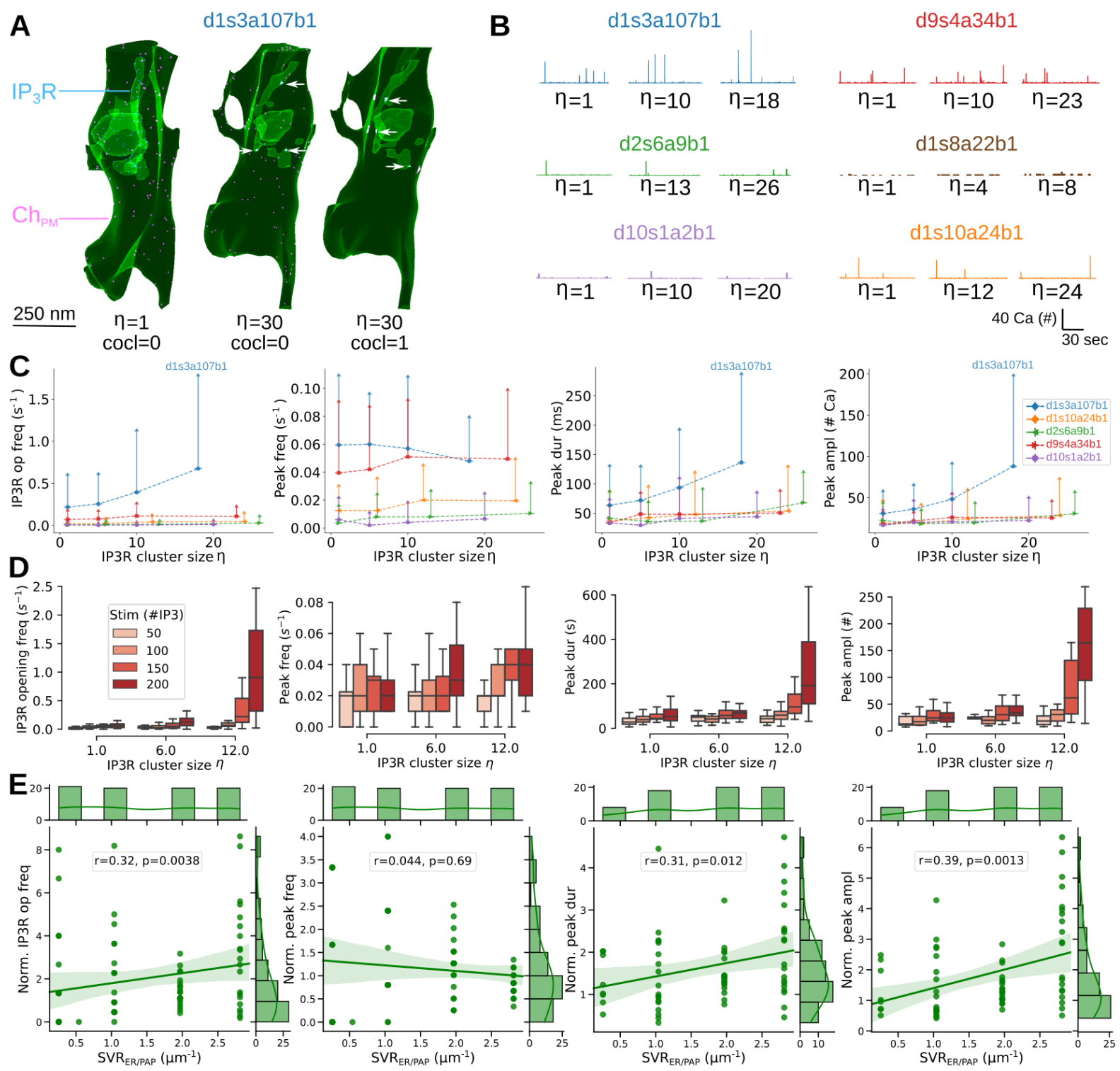

Fig 5: The effect of IP3R clustering differs from PAP to PAP. (A) Screenshots of simulations in $\mathrm{d} 1 \mathrm{~s} 3 \mathrm{a} 107 \mathrm{~b} 1$, with $\mathrm{IP}_{3} \mathrm{R}$ cluster size $\eta=1$ and $\eta=30$. Simulations were performed with (cocl=1) and without (cocl=0) co-clustering of $\mathrm{Ca}^{2+}$ channels at the plasma membrane (purple) with $\mathrm{IP}_{3}$ Rs on the ER (blue). $\mathrm{IP}_{3} \mathrm{R}$ clusters are indicated with a white arrow. (B) Representative free $\mathrm{Ca}^{2+}$ traces measured in silico in d1s3a107b1 (blue), d9s4a34b1 (red), d2s6a9b1 (green), d1s8a22b1 (brown), d10s1a2b1 (purple) and d1s10a24b1 (orange) meshes. (C) Quantification of $\mathrm{IP}_{3} \mathrm{R}$ opening frequency (left), $\mathrm{Ca}^{2+}$ peak frequency (middle left), duration (middle right) and amplitude (right) as a function of $\eta$, in d1s3a107b1 (blue circle), d1s10a24b1 (orange diamond), d2s6a9b1 (green triangle), d9s4a34b1 (red triangle) and d10s1a2b1 (purple hexagon), cocl $=1$. Data are represented as mean $\pm \mathrm{STD}, \mathrm{n}=20$ for each mesh and cluster size tested. 
Fig 5: Lines are guides for the eyes. Note that no peaks were detected in simulations in d1s8a22b1. (D) Quantification of $\mathrm{IP}_{3} \mathrm{R}$ opening frequency (left), $\mathrm{Ca}^{2+}$ peak frequency (middle left), duration (middle right) and amplitude (right), in d1s10a24b1, for $\eta=1,6$ and 12 and various levels of neuronal stimulation: $\mathrm{IP}_{3}$ infused $\mathrm{i}=50,100,150$ and 200 (from left to right, light red to dark red), $n=20$ for each parameter set tested. (E) Scatterplots presenting the variation of $\mathrm{IP}_{3} \mathrm{R}$ opening frequency (left), $\mathrm{Ca}^{2+}$ peak frequency (middle left), duration (middle right) and amplitude (right) for $\eta=18-24$, normalized with $\mathrm{Ca}^{2+}$ peak characteristics measured for $\eta=1$, as a function of $\mathrm{SVR}_{\mathrm{ER} / \mathrm{PAP}}$. Plots are accompanied by univariate kernel density estimation curves and a linear regression fit. Spearman correlation coefficient, $r$, and $p$-value, $p$, are displayed onto each regression plot.

PAPs could act as an amplifier of neuronal stimulation.

Unexpectedly, the PAPs in which an $\mathrm{IP}_{3} \mathrm{R}$ clustering effect was observed were not the PAPs with the highest ER surface area $\mathrm{S}_{\mathrm{ER}}$, i.e with the highest number of $\mathrm{IP}_{3} \mathrm{R}$ channels. Indeed, although the normalized $\mathrm{IP}_{3} \mathrm{R}$ opening frequency and $\mathrm{Ca}^{2+}$ peak frequency at high cluster size were positively correlated with $\mathrm{S}_{\mathrm{ER}}$, normalized $\mathrm{Ca}^{2+}$ peak amplitude and duration were not correlated with $\mathrm{S}_{\mathrm{ER}}$ (Supplemental Fig. S2). Rather, normalized $\mathrm{IP}_{3} \mathrm{R}$ opening frequency, $\mathrm{Ca}^{2+}$ peak amplitude and duration were positively correlated to the ratio between ER surface area and PAP volume $\mathrm{SVR}_{\mathrm{ER} / \mathrm{PAP}}$ (Fig. 5E, p=0.0038, 0.012 and 0.0013 , respectively). $\mathrm{Ca}^{2+}$ peak frequency however did not vary with $\mathrm{SVR}_{\mathrm{ER} / \mathrm{PAP}}($ Fig. $5 \mathrm{E}, \mathrm{p}=0.69)$. This probably results from our peak definition. Indeed, as a peak is considered terminated when the $\mathrm{Ca}^{2+}$ trace decreases below peak threshold, a higher frequency of $\mathrm{IP}_{3} \mathrm{R}$ opening events can result in successive opening events occurring before peak termination, resulting in a similar peak frequency but larger peak duration.

Overall, our simulation results nuance the effect of the clustering of $\mathrm{Ca}^{2+}$ channels on $\mathrm{Ca}^{2+}$ signals in small sub-cellular compartments like PAPs. Strikingly, in contrast with reports from models in 2 spatial dimensions $(57,59), \mathrm{Ca}^{2+}$ activity increased with cluster size in only a subset of the realistic 3D PAP meshes tested, highlighting the complex interplay between the ER surface to PAP volume ratio, the intensity of neuronal stimulation and $\mathrm{IP}_{3} \mathrm{R}$ clustering on $\mathrm{Ca}^{2+}$ microdomain activity. This highlights the importance of cautious interpretation of simulation results on geometrical effects depending on the geometry used.

\section{ER SVR conditions the amplification of $\mathrm{Ca}^{2+}$ activity by $\mathrm{IP}_{3} \mathbf{R}$ clustering in 3D}

Our simulation results revealed that the increase of $\mathrm{Ca}^{2+}$ activity resulting from $\mathrm{IP}_{3} \mathrm{R}$ clustering increased with the ratio between the ER surface area and the PAP volume $\left(\mathrm{SVR}_{\mathrm{ER} / \mathrm{PAP}}\right.$, Fig $5 E$ ). In the PAP meshes studied in Fig. $5, \mathrm{SVR}_{\mathrm{ER} / \mathrm{PAP}}$ varied together with PAP shape and ER 
shape. To discern the effect of ER and PAP shape from $\mathrm{SVR}_{\mathrm{ER} / \mathrm{PAP}}$ on $\mathrm{Ca}^{2+}$ activity in PAPs, we created meshes with various ER size and constant ER and PAP shapes. The original mesh was extracted from the $220 \mu \mathrm{m}^{3}$ astrocytic volume, located at the vicinity of the $\mathrm{d} 9 \mathrm{~s} 3 \mathrm{a} 51 \mathrm{~b} 1$ PSD and referred to as PAP1. The location of PAP1 in the $220 \mu \mathrm{m}^{3}$ reconstructed hippocampal astrocytic volume is presented in Supplemental Fig. S3. Meshes with various SVRER/PAP were created from PAP1 by rescaling the ER using Blender software. Meshes were then created following the mesh pre-processing workflow described in Fig. 4C, resulting in the creation of $P A P 1_{v}, P A P 1_{w}, P A P 1_{x}, P A P 1_{y}$ and $P A P 1_{z}$ meshes (Fig. 6A). The geometrical properties of those meshes are presented in Table 1.

$\mathrm{IP}_{3} \mathrm{R}$ opening frequency, $\mathrm{Ca}^{2+}$ peak frequency, duration and amplitude increased with $\mathrm{SVR}_{\mathrm{ER} / \mathrm{PAP}}$ (Fig. 6B-F). This is not surprising as ER surface area increases with $\mathrm{SVR}_{\mathrm{ER} / \mathrm{PAP}}$ in those meshes, thus resulting in an increase of the amount of $\mathrm{IP}_{3} \mathrm{R}$ channels with $\mathrm{SVR}_{\mathrm{ER} / \mathrm{PAP}}$. The total number of $\mathrm{IP}_{3} \mathrm{R}$ channels, $N_{\mathrm{IP} 3 \mathrm{R}}$, thus was $24,120,240,360$ and 460 , in $\mathrm{PAP} 1_{\mathrm{v}}$, $\mathrm{PAP} 1_{\mathrm{w}}, \mathrm{PAP} 1_{\mathrm{x}}, \mathrm{PAP} 1_{\mathrm{y}}$ and $\mathrm{PAP} 1_{\mathrm{z}}$ meshes, respectively. Importantly, $\mathrm{Ca}^{2+}$ peak frequency (Fig. 6D, ANOVA, $\mathrm{p}=2.39 e^{-8}$ ), duration (Fig. 6E, ANOVA, $\mathrm{p}=7.52 e^{-17}$ ) and amplitude (Fig. $6 \mathrm{~F}$, ANOVA, $\mathrm{p}=1.29 e^{-14}$ ) increased with $\mathrm{IP}_{3} \mathrm{R}$ cluster size in $\mathrm{PAP} 1_{\mathrm{z}}$ mesh. This resulted from an increase of $\mathrm{IP}_{3} \mathrm{R}$ opening frequency with cluster size in $\mathrm{PAP} 1_{\mathrm{z}}$ (Fig. 6C, ANOVA, $\mathrm{p}=5.93 e^{-24}$ ), No clustering effect was observed in $\mathrm{PAP} 1_{\mathrm{w}-\mathrm{y}}$ meshes, characterized by a lower $\mathrm{SVR}_{\mathrm{ER} / \mathrm{PAP}}$, confirming the mechanistic link between $\mathrm{SVR}_{\mathrm{ER} / \mathrm{PAP}}$ and the amplification of $\mathrm{Ca}^{2+}$ activity mediated by $\mathrm{IP}_{3} \mathrm{R}$ clustering suggested in Fig. 5. Note that no $\mathrm{Ca}^{2+}$ signals were detected in $\mathrm{PAP} 1_{\mathrm{v}}$ mesh. Simulations in $\mathrm{PAP} 1_{\mathrm{z}}$ meshes with constant $\mathrm{IP}_{3} \mathrm{R}$ channel number further highlight that this effect is mediated by $\mathrm{SVR}_{\mathrm{ER} / \mathrm{PAP}}$ rather than by $\mathrm{IP}_{3} \mathrm{R}$ channel density (Supplemental Fig. S4). Supplemental Fig. S5 reveals that $\mathrm{IP}_{3} \mathrm{R}$ opening frequency and $\mathrm{Ca}^{2+}$ peak frequency increased with $\mathrm{SVR}_{\mathrm{ER} / \mathrm{PAP}}$ even when $\mathrm{IP}_{3} \mathrm{R}$ channels were not clustered $(\eta=1)$. Increasing ER surface area in PAP1 however also resulted in a decreased distance between the ER and the plasma membrane (PM) in the PAP (Fig. 6G). Importantly, $\mathrm{Ca}^{2+}$ peak frequency

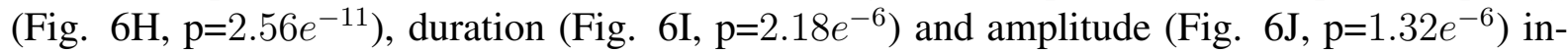
creased with the amount of ER vertices at ER-PM contact sites $(\leq 20 \mathrm{~nm}$ to the closest PM vertex $(60,61)$ ). This suggests that the increased $\mathrm{IP}_{3} \mathrm{R}$ clustering effect on $\mathrm{Ca}^{2+}$ microdomain activity in $\mathrm{PAP}_{2}$ could result either from its increased $\mathrm{SVR}_{\mathrm{ER} / \mathrm{PAP}}$ or to the decreased ER-PM distances in this mesh compared to PAP $1_{w-y}$ meshes. Overall, our simulation results suggest that ER shape, notably the ratio between its surface area and PAP volume, shape astrocytic $\mathrm{Ca}^{2+}$ microdomain activity at synapses.

\section{Quantification of ER-PM distance distribution within PAPs}

As simulation results suggested that $\mathrm{Ca}^{2+}$ activity in $\mathrm{PAP} 1_{\mathrm{v}-\mathrm{z}}$ meshes varies depending on the distribution of the ER within the PAP, we next aimed at quantifying ER distribution in the 21 ER-containing PAP meshes reconstructed from EM and presented in Fig. 3. To do so, we measured the distance between each vertex on the plasma membrane (PM) and the closest 


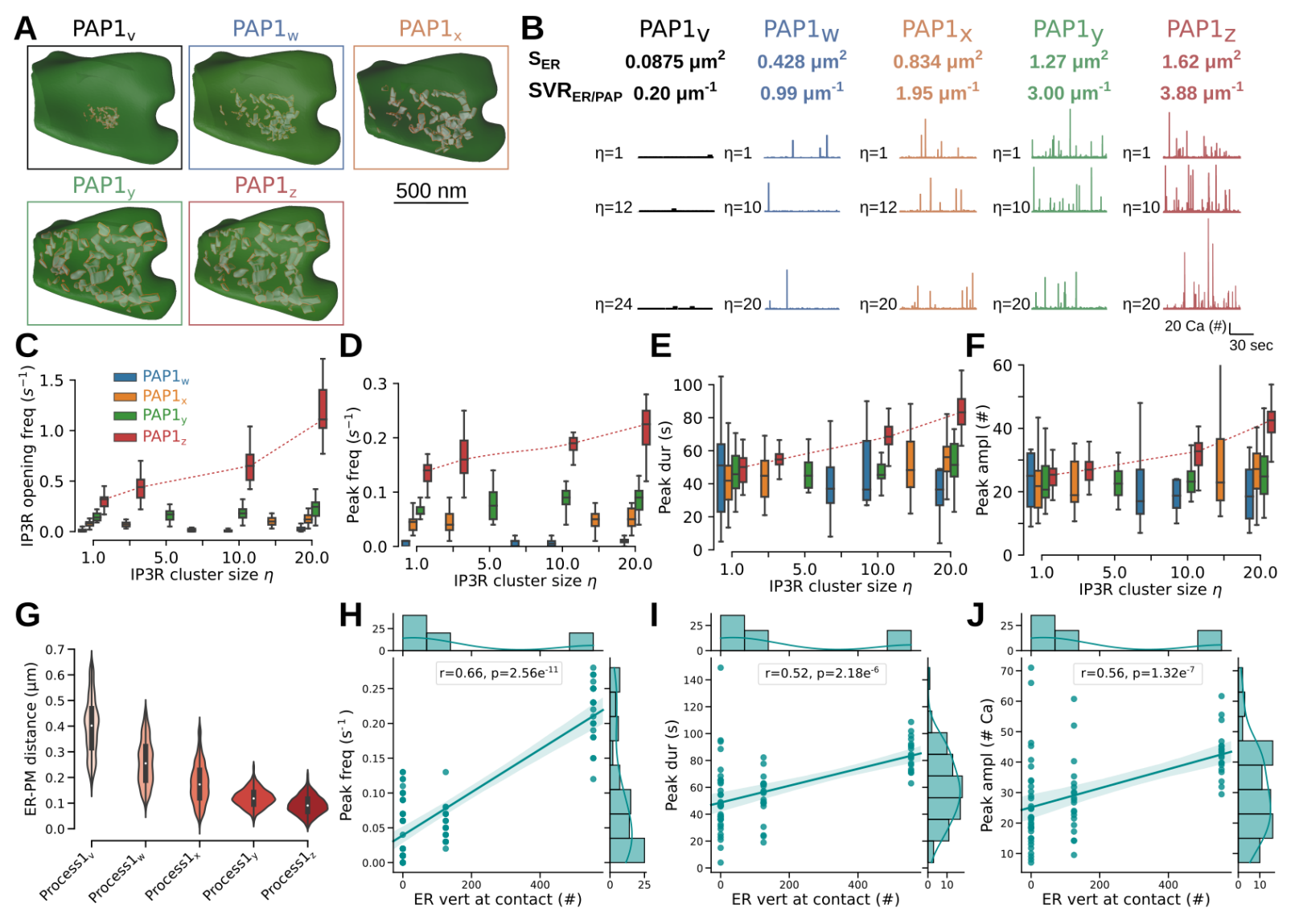

Fig 6: The surface-volume ratio of the ER in PAPs conditions the amplification of $\mathrm{Ca}^{2+}$ activity by $\mathrm{IP}_{3} \mathbf{R}$ clustering in 3D. (A) Images of the different PAP meshes created to investigate the effect of the ratio between ER surface area and PAP volume, $\mathrm{SVR}_{\mathrm{ER} / \mathrm{PAP}}$, on $\mathrm{Ca}^{2+}$ microdomain activity: $\mathrm{PAP} 1_{\mathrm{v}-\mathrm{z}}$. Meshes were obtained by rescaling the ER object in PAP1, located at the vicinity of the d9s3a51b1 PSD (Supplemental Fig. S3). Geometrical features of the meshes are presented in Table 1. (B) Representative free $\mathrm{Ca}^{2+}$ traces measured in PAP1 $1_{\mathrm{V}}$ (black), $\mathrm{PAP} 1_{\mathrm{w}}$ (blue), $\mathrm{PAP} 1_{\mathrm{x}}$ (orange), $\mathrm{PAP} 1_{\mathrm{y}}$ (green) and $\mathrm{PAP} 1_{\mathrm{z}}$ (red), for $\mathrm{IP}_{3} \mathrm{R}$ cluster size $\eta=1$ (top), $\eta=12$ (middle) and $\eta=24$ (bottom). (C-F) Quantification of $\mathrm{IP}_{3} \mathrm{R}$ opening frequency (C), $\mathrm{Ca}^{2+}$ peak frequency (D), duration (E) and amplitude $(\mathrm{F})$, in $\mathrm{PAP} 1_{\mathrm{w}}$ (blue), $\mathrm{PAP} 1_{\mathrm{x}}$ (orange), $P A P 1_{\mathrm{y}}$ (green) and $\mathrm{PAP} 1_{\mathrm{z}}$ (red), for $\eta=1-23$. Note that $\eta$ varies depending on the mesh (see Methods). Lines were added to visualize the effect of $\eta$ on $\mathrm{Ca}^{2+}$ peak characteristics in $\mathrm{PAP}_{\mathrm{z}}$ mesh. (G) Quantification of the variation of the distance between each ER vertex and the closest plasma membrane $(\mathrm{PM})$ vertex in $P A P 1_{\mathrm{v}-\mathrm{z}}$ meshes. (H-J) Scatterplots presenting $\mathrm{Ca}^{2+}$ peak frequency $(\mathrm{H})$, duration $(\mathrm{I})$ and amplitude $(\mathrm{J})$ in $\mathrm{PAP} 1_{\mathrm{v}-\mathrm{z}}$ meshes for $\eta=20$, normalized with $\mathrm{Ca}^{2+}$ peak characteristics for $\eta=1$, as a function of the number of ER vertices $\leq 20$ $\mathrm{nm}$ to the closest PM vertex. 
Fig 6: Plots are presented with univariate kernel density estimation curves and a linear regression fit. Spearman correlation coefficient, $r$, and $p$-value, $p$, are displayed onto each regression plot.

vertex on the ER. We found that ER-PM distance is highly variable in PAPs from a single cell, with an average ER-PM distance within a single PAP from around $200 \mathrm{~nm}$ to $1200 \mathrm{~nm}$ (Fig. 7B-C). Not surprisingly, mean ER-PM distance decreases as ER (Fig. 7D, $\mathrm{p}=5.01 e^{-10}$ ) and PAP (Fig. 7E, $\mathrm{p}=0.055$ ) surface area increase. Interestingly, ER-PM distance was lower in PAPs contacting boutons with higher surface area (Fig. 7F, $\mathrm{p}=0.022$ ). Note that there was no correlation between ER-PM distance and spine surface area (Fig. $7 \mathrm{G}, \mathrm{p}=0.73$ ). Importantly, we found that PAPs closer to the synapse are characterized by lower mean ER-PM distance (Fig. $7 \mathrm{H}, \mathrm{p}=2.2 e^{-5}$ ), which, according to simulation results presented in Fig. 6, could result in enhanced $\mathrm{Ca}^{2+}$ activity in those PAPs.

\section{Effect of ER-PM distance in PAPs on $\mathrm{Ca}^{2+}$ microdomain activity}

To discern the effect of SVR $\mathrm{ER} / \mathrm{PAP}$ from the effect of ER-PM distance on $\mathrm{Ca}^{2+}$ microdomain activity in PAPs reported in Fig. 6, we implemented an algorithm that creates realistic tetrahedral 3D meshes of PAPs characterized by various distributions of the ER within the same PAP. The workflow is presented in Fig. 8. Briefly, the ER is split into small portions of similar size, then resized to match the total ER surface area of the original mesh. Simulations in meshes with the original ER and with split ER confirmed that this ER splitting algorithm does not alter $\mathrm{Ca}^{2+}$ activity in the PAP (Supplemental Fig. S6). A simulation of $\mathrm{n}$ frames is then generated in Blender, which alters the location of the ER objects within the PAP. Each frame is thus characterized by a unique distribution of the ER objects within the PAP, while ER and PAP shape, surface area, volume and SVR are constant across frames (Supplemental movie 2). The mesh processing workflow presented in Fig. $4 \mathrm{C}$ is then automatically applied to each frame of interest. This workflow allows the creation of numerous realistic 3D PAP meshes, that can be used for reaction-diffusion simulations in 3D. Fig. 7B-D displays the quantification of ER distribution in the PAP meshes created with this workflow on PAP d1s15a32b1. The workflow successfully produced realistic tetrahedral PAP meshes characterized by various ER-PM distances (Fig. 7B). Note that the distribution of ER-PM distance at ER-PM contact sites (ER-PM distance $\leq 20 \mathrm{~nm}$ ) did not vary, while the number of ER vertices belonging to contact sites, and thus the size of the contact sites, increased with frame number (Fig. 7C).

To test the effect of ER distribution in PAPs, simulations were performed in meshes from frames $0,21,64$ and 250 of PAP d1s15a32b1 (Fig. 9A) with different $\mathrm{IP}_{3} \mathrm{R}$ cluster sizes, $\eta$. Free $\mathrm{Ca}^{2+}$ signals did not vary depending on ER distribution within the PAP (Fig. 9C). Interestingly, ER distribution did not affect the amplification of $\mathrm{IP}_{3} \mathrm{R}$ opening frequency (ANOVA, $\mathrm{p}=0.059$ ), $\mathrm{Ca}^{2+}$ peak duration (ANOVA, $\mathrm{p}=0.55$ ) and amplitude (ANOVA, $\mathrm{p}=0.15$ ) with $\mathrm{IP}_{3} \mathrm{R}$ 


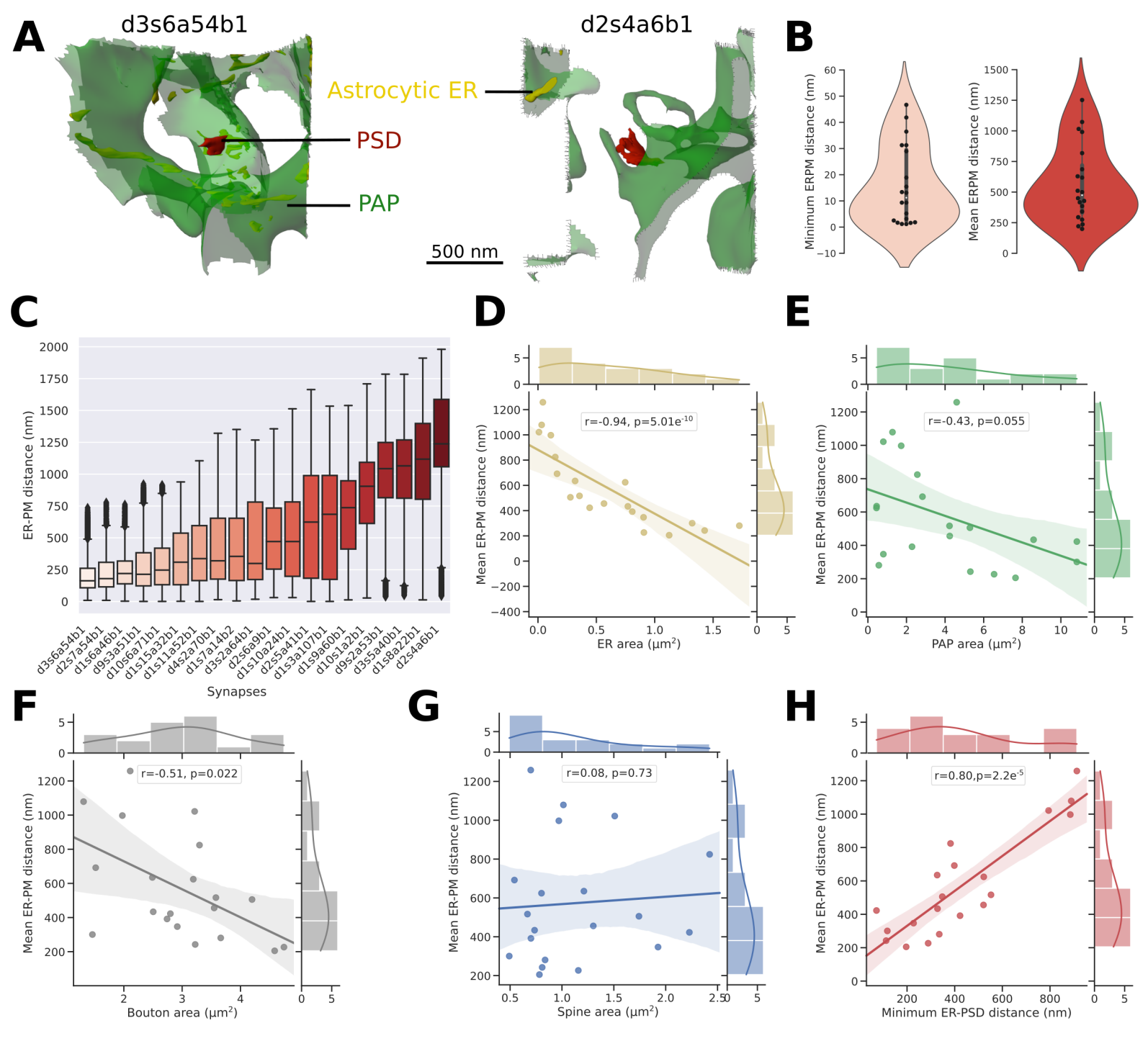

Fig 7: ER-PM distance in PAPs is highly variable and is decreased in PAPs close to the synapse. (A) Images of 2 PAP meshes (green), d3s6a54b1 (left) and d2s4a6b1 (right) with the neighboring PSD (red), displaying the diverse shapes and distributions of the ER (yellow) in PAPs from the same cell. (B) Distribution of the minimum (left) and mean (right) distance between each vertex on the plasma membrane (PM) and the closest ER vertex, measured in PAP meshes reconstructed from EM, n=21 (Fig. 3). (C) Quantification of the distance between each PM vertex and the closest ER vertex in each PAP mesh. (D-F) Scatterplots presenting the variation of the mean distance between each PM vertex and the closest ER vertex as a function of ER (D), PAP (E), bouton (F), spine (G) surface area and as a function of the minimum ERPSD distance $(H), n=21$. Plots are presented with univariate kernel density estimation curves and a linear regression fit. Spearman correlation coefficient, $r$, and $p$-value, $p$, are displayed onto each regression plot. 

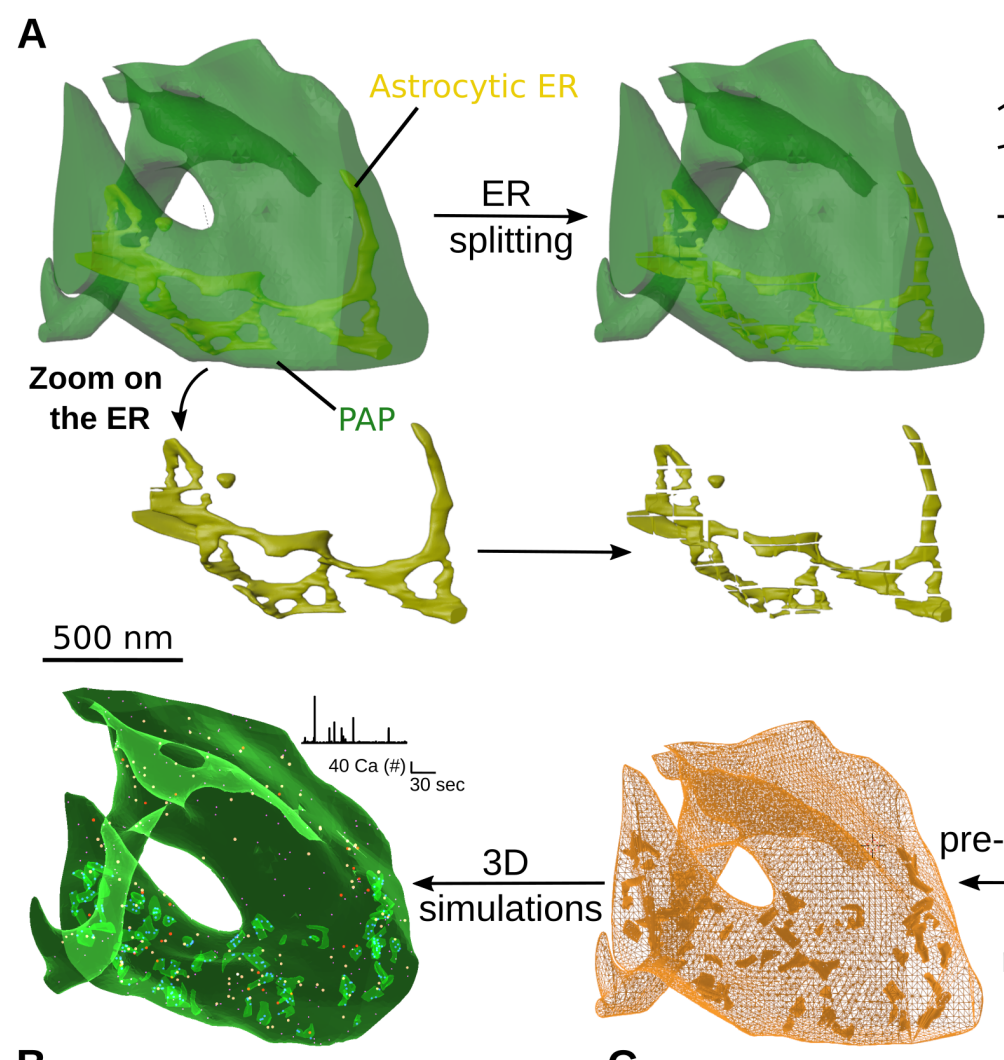

B

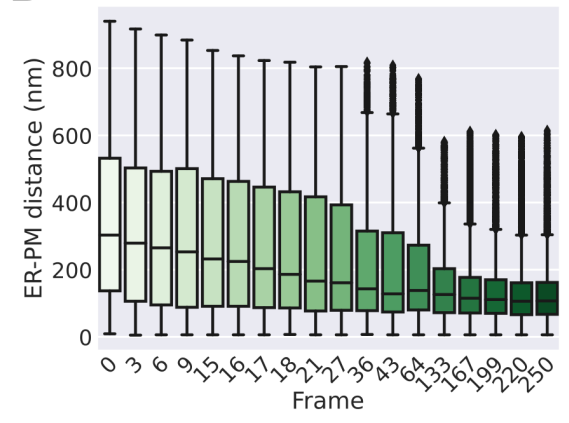

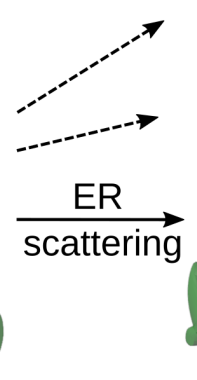
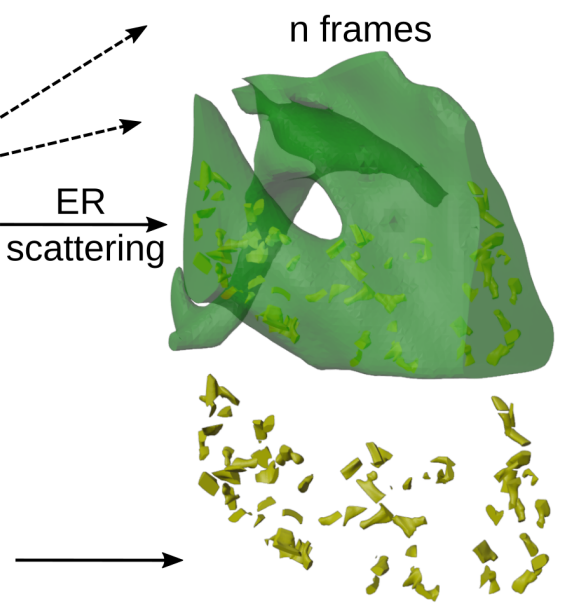

ER-PM detection \& contact sites $\sqrt{\text { quantification }}$

Fig 8: Automated realistic 3D PAP mesh generation with diverse ER distributions. (A) Schematic representing the workflow developed in this study to create realistic PAP meshes in 3 spatial dimensions with various ER distributions and constant shape, volume and surface area of PAP and ER, used on the PAP mesh d1s15a32b1. The ER is split and a simulation with $n$ frames is generated in Blender, in which ER objects are subject to physical forces that alter their spatial distribution. The $\mathrm{n}$ frames are thus characterized by different locations of the ER elements within the PAP, with constant ER and PAP shapes. The pipeline detects, quantifies and exports in a text file the distance between each vertex at the plasma membrane (PM) and the closest vertex at the membrane of the ER. A point cloud can be created to visualize the ER vertices at ER-PM contact sites (ER-PM distance $\leq 20 \mathrm{~nm}$, white arrows). The mesh preprocessing workflow presented in Fig. 4C is then applied to the mesh of each desired frame. The resulting $3 \mathrm{D}$ tetrahedral meshes can then be used for $3 \mathrm{D}$ reaction-diffusion simulations. 
Fig 8: (B) Quantification of the distance between each PM vertex and the closest ER vertex in the meshes generated by the workflow presented in panel A, applied to the d1s15a32b1 PAP mesh. (C) Quantification of the number of ER vertices located at ER-PM contact sites, i.e $\leq$ $20 \mathrm{~nm}$ to the closest PM vertex, in each frame from mesh d1s15a32b1. (D) Quantification of the distance between each PM vertex and the closest ER vertex at ER-PM contact sites, in each frame from mesh d1s15a32b1.

cluster size when $\mathrm{IP}_{3} \mathrm{R}$ clusters were randomly distributed on the membrane of the ER (Fig. 9D). However, the increase of peak frequency with cluster size was larger in meshes in which the ER was closer to the plasma membrane (Fig 9D, ANOVA, p=0.0048). This suggests that a distribution of the ER closer to the plasma membrane might allow increased $\mathrm{Ca}^{2+}$ peak frequency with $\mathrm{IP}_{3} \mathrm{R}$ cluster size.

As $\mathrm{IP}_{3} \mathrm{R}$ clusters are believed to be preferentially located at ER-PM contact sites (54), we performed simulations in the same meshes while positioning $\mathrm{IP}_{3} \mathrm{R}$ clusters at ER-PM contact sites. Screenshots of simulations with different locations of $\mathrm{IP}_{3} \mathrm{R}$ clusters are presented in Fig. 9B. Interestingly, locating $\mathrm{IP}_{3} \mathrm{R}$ clusters at ER-PM contact sites resulted in larger increases of $\mathrm{IP}_{3} \mathrm{R}$ opening frequency (ANOVA, $\mathrm{p}=0.0019$ ) and $\mathrm{Ca}^{2+}$ peak frequency (ANOVA, $\mathrm{p}=0.0043$ ) with cluster size in meshes in which the ER was closer to the PM (Fig. 9D). However, the location of the ER did not impact the effect of $\mathrm{IP}_{3} \mathrm{R}$ cluster size on $\mathrm{Ca}^{2+}$ peak duration (ANOVA, $\mathrm{p}=0.45$ ) and amplitude (ANOVA, $\mathrm{p}=0.069$ ). Together, those results suggest that a distribution of the ER closer to the plasma membrane, coupled with a location of $\mathrm{IP}_{3} \mathrm{R}$ clusters at ER-PM contact sites, favors an increase of $\mathrm{Ca}^{2+}$ peak frequency with cluster size. ER-PM contact sites could act as diffusional barriers. Locating $\mathrm{IP}_{3} \mathrm{R}$ channels at ER-PM contact sites would thus increase the residency time of $\mathrm{Ca}^{2+}$ ions and $\mathrm{IP}_{3}$ molecules at the vicinity of the channels, thus increasing the probability of $\mathrm{Ca}^{2+}$ and $\mathrm{IP}_{3}$ binding to $\mathrm{IP}_{3} \mathrm{Rs}$ and resulting in an increased $\mathrm{IP}_{3} \mathrm{R}$ opening frequency in meshes with larger ER-PM contact sites. To further test the interplay between ER distribution, $\mathrm{IP}_{3} \mathrm{R}$ clustering and local diffusional properties, $\mathrm{Ca}^{2+}$ buffers, here $\mathrm{Ca}^{2+}$ indicators GCaMP6s, were added to the model. Strikingly, ER distribution had a greater effect on buffered $\mathrm{Ca}^{2+}$ signals compared to free $\mathrm{Ca}^{2+}$ signals (Fig. 9E). More precisely, the increase of $\mathrm{IP}_{3} \mathrm{R}$ opening frequency (ANOVA, $\mathrm{p}=2.37 e^{-7}$ ), Ca-GCaMP peak frequency (ANOVA, $\mathrm{p}=1.14 e^{-4}$ ), duration (ANOVA, $\mathrm{p}=7.78 e^{-3}$ ) and amplitude (ANOVA, $\mathrm{p}=0.023$ ) with $\mathrm{IP}_{3} \mathrm{R}$ cluster size was significantly larger in meshes in which the ER was located closer to the plasma membrane. Those results highlight that the effect of ER distribution within the PAP on $\mathrm{Ca}^{2+}$ microdomain activity is conditioned by the location of $\mathrm{IP}_{3} \mathrm{R}$ channels at ER-PM contact sites and by local $\mathrm{Ca}^{2+}$ buffering. 

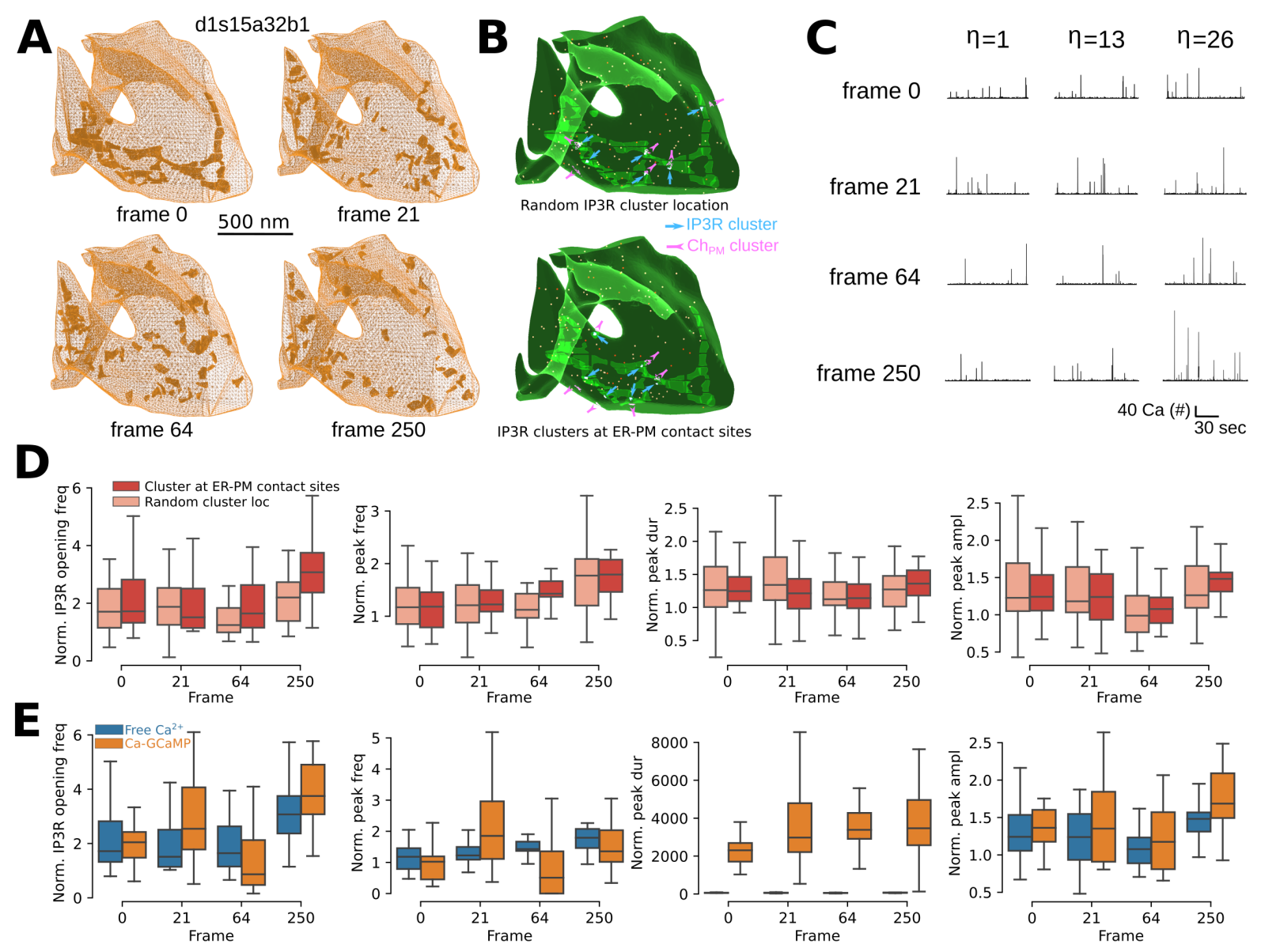

Fig 9: $\mathrm{Ca}^{2+}$ buffering and $\mathrm{IP}_{3} \mathbf{R}$ channel location mediate the effect of ER-PM distance on $\mathrm{Ca}^{2+}$ microdomain activity in PAPs. (A) Images presenting different meshes created from PAP d1s15a32b1 using the automated workflow presented in Fig. 8: frames 0, 21, 64 and 250, characterized by diverse ER distributions within the PAP with constant PAP and ER shape, volume and surface area. Characteristics of ER-PM distance in those meshes are displayed in Fig. 8B-D. (B) Screenshots of simulations performed in $\mathrm{d} 1 \mathrm{~s} 15 \mathrm{a} 32 \mathrm{~b} 1_{\mathrm{f} 0}$ mesh (frame 0 ), with $\mathrm{IP}_{3} \mathrm{R}$ clusters (blue, arrows) distributed randomly on the ER membrane (top) or at ER-PM contact sites (bottom). $\mathrm{IP}_{3} \mathrm{R}$ clusters were co-localized with $C h_{\mathrm{PM}}$ clusters at the plasma membrane (purple, inverse arrows): cocl=1. (C) Representative free $\mathrm{Ca}^{2+}$ traces in frames $0,21,64$ and 250 , with $\mathrm{IP}_{3} \mathrm{R}$ cluster size $\eta=1,13$ and 26 and random distribution of $\mathrm{IP}_{3} \mathrm{R}$ clusters on the ER. (D) Quantification of $\mathrm{IP}_{3} \mathrm{R}$ opening frequency (left), $\mathrm{Ca}^{2+}$ peak frequency (middle left), duration (middle right) and amplitude (right), in frames 0, 21, 64 and 250, for $\eta=26$, normalized by $\mathrm{Ca}^{2+}$ peak characteristics for $\eta=1$ in each mesh, with $\mathrm{IP}_{3} \mathrm{R}$ clusters at random locations on the ER (left, light red) or at ER-PM contact sites (right, dark red). 
Fig 9: (E) Quantification of $\mathrm{IP}_{3} \mathrm{R}$ opening frequency (left), peak frequency (middle left), duration (middle right) and amplitude (right) of free $\mathrm{Ca}^{2+}$ signals (blue) and Ca-GCaMP signals (orange), in frames $0,21,64$ and 250 , for $\eta=26$, normalized by $\mathrm{Ca}^{2+}$ peak characteristics for $\eta=1$. $\mathrm{IP}_{3} \mathrm{R}$ clusters were located at ER-PM contact sites. $\mathrm{n}=20$ for each parameter set tested.

\section{Discussion}

Here, we extracted 3D meshes of tripartite synapses from a $220 \mu \mathrm{m}^{3}$ hippocampal astrocytic volume from the CA1 stratum radiatum region, reconstructed from EM (47). Quantification of the geometrical features of those meshes highlighted the diverse geometrical properties of PAPs from a single astrocyte and revealed, contrary to a widespread belief that PAPs are devoid of ER $(23,44)$, that $75 \%$ of PAPs contained some ER. Interestingly, we found that PAPs are the closest to the synapse when bouton surface area is low, which could result from the spatial constraints imposed by larger boutons, preventing the PAP from getting in close contact to the PSD. Reaction-diffusion simulations in the realistic PAP 3D meshes reconstructed in this study provided key insights into the effect of the diverse shapes and distributions of the ER in PAPs on microdomain $\mathrm{Ca}^{2+}$ activity. As reactive astrocytes, hallmark of brain diseases (62), are characterized by a remodelling of ER volume and shape (63), our results suggest that such geometrical alterations of the ER could be one of the factors responsible for the altered astrocytic $\mathrm{Ca}^{2+}$ activity reported in pathological conditions (64).

Fine-tuning the spatial distribution of $\mathrm{Ca}^{2+}$ channels, monitoring channel opening events at each channel, while independently manipulating ER shape and distribution, such as performed in this study, is not feasible experimentally. It is yet essential to understand the mechanistic link between the spatial features of the astrocyte and its $\mathrm{Ca}^{2+}$ microdomain activity. Combining our detailed biophysical model of $\mathrm{Ca}^{2+}$ signals in PAPs, the PAP meshes that we extracted from EM and the realistic PAP meshes with various ER distributions generated by our automated mesh generator allowed us to provide key insights into $\mathrm{Ca}^{2+}$ signaling in PAPs. Notably, we predict how the complex interplay between the clustering of $\mathrm{Ca}^{2+}$ channels, the ratio between ER surface surface area and PAP volume, $\mathrm{Ca}^{2+}$ buffering and the size and location of ER-PM contact sites shapes $\mathrm{Ca}^{2+}$ microdomain signals at tripartite synapses. This study is the first to our knowledge to model $\mathrm{Ca}^{2+}$ activity in astrocytes with realistic shapes in 3D at the nanoscale that accounts for the complex and diverse spatial characteristics of $\mathrm{Ca}^{2+}$ stores in PAPs. Furthermore, our results highlight the impact of the modeling choices on simulation results, notably when investigating spatial effects. Importantly, our results nuance the effect of the clustering of $\mathrm{Ca}^{2+}$ channels, which is stronger in $2 \mathrm{D}$ or simple 3D shapes than in more realistic 3D meshes. This is crucial as, until now, modeling studies on PAPs were conducted in 1D, 2D or in simple 3D shapes, notably cylinders $(49,65-69)$. The 3D meshes provided by this study, together with our realistic 3D PAP mesh generator, pave the way for future modeling studies in realistic 3D meshes to investigate the mechanisms governing neuron-astrocyte communication at tripartite 
synapses.

The geometrical data used here were extracted from electron microscopy, which is the only tool that can resolve PAP and ER shape at a high spatial resolution (6 $\mathrm{nm}$ here), yet results in potential alterations of the ultrastructure of the extracellular space (70) and cannot be used to study live cells. Furthermore, the model used in this study, focusing on the effect of the ER shape and distribution on $\mathrm{Ca}^{2+}$ activity, describes with great details the kinetics of ERmediated $\mathrm{Ca}^{2+}$ signals while simplifying other $\mathrm{Ca}^{2+}$ sources. Other $\mathrm{Ca}^{2+}$ sources and channels however contribute to $\mathrm{Ca}^{2+}$ microdomain activity in PAPs, including mitochondria, the $\mathrm{Na}^{+} / \mathrm{Ca}^{2+}$ exchanger, transient receptor potential ankyrin 1 channels, L-type voltage gated channels and other pathways $(27,43)$. According to our model's predictions, the spatial distribution of $\mathrm{Ca}^{2+}$ channels can alter the spatio-temporal properties of $\mathrm{Ca}^{2+}$ microdomain signals in PAPs as well as their amplification upon neuronal stimulation. Further quantification of the $\mathrm{Ca}^{2+}$ channels expressed in PAPs, their density, location and remodeling in live tissue under (patho-)physiological conditions is thus essential to better understand astrocyte activity at synapses. The recent advances in super-resolution techniques, notably single particle tracking methods, provide a promising avenue to overcome current limitations in obtaining such data $(21,71)$.

Recent super-resolution studies in live neurons revealed dynamical remodeling of ER-PM contact sites (72) and diffusional trapping of molecules resulting from the ER remodeling (73) in neurons. Those observations, together with our model predictions, highlight the need for further quantification of the dynamical shape and distribution of the ER in astrocytes in live tissue to fully grasp its influence on $\mathrm{Ca}^{2+}$ microdomain activity in astroytes. According to our model predictions, preferential location of $\mathrm{IP}_{3} \mathrm{Rs}$ at ER-PM contact sites might be essential to allow signal amplification with $\mathrm{IP}_{3} \mathrm{R}$ cluster size and could thus strongly alter the spatio-temporal properties of astrocytic $\mathrm{Ca}^{2+}$ signals evoked by neurotransmitters, potentially affecting the subsequent modulation of neuronal activity by astrocytes. Our results, in accordance with previous computational studies in other cell types (52), highlight that $\mathrm{Ca}^{2+}$ buffering plays a crucial role in shaping $\mathrm{Ca}^{2+}$ activity at ER-PM contact sites. The $\mathrm{Ca}^{2+}$ buffering effect described here was mediated by $\mathrm{Ca}^{2+}$ indicators. Future experimental and computational studies will be essential to assess $\mathrm{Ca}^{2+}$ buffering mechanisms in astrocytes and PAPs, which are still poorly understood, yet, according to our simulation results, play crucial roles in shaping $\mathrm{Ca}^{2+}$ microdomain activity in astrocytes.

Overall, this study provides new insights into astrocytic activity at tripartite synapses by characterizing the presence, shape and distribution of the ER in PAPs and by shedding light to the mechanistic link between those features and microdomain $\mathrm{Ca}^{2+}$ activity at tripartite synapses. The realistic 3D meshes of tripartite synapses created in this study pave the way for new modeling studies of neuron-astrocyte communication in the synaptic micro-environment, allowing the study of various processes, such as glutamate spillover or gliotransmission. Such 
studies will be crucial to decipher whether the various nano-architectures displayed by tripartite synapses reflect distinct functional identities.

\section{Materials and Methods}

\section{D reconstruction from electron microscopy}

\section{Sample preparation and imaging}

The original dataset used in this work (EM stack and 3D reconstructions) was previously published in (47). The block was a gift from Graham Knott (BioEM imaging facility at EPFL, Lausanne, Switzerland). All procedures were performed according to the Swiss Federal Laws. One P90 Sprague-Dawley rat was deeply anesthetized with isoflurane and transcardially perfused using 2\% paraformaldehyde and $2.5 \%$ glutaraldehyde in PBS $0.1 \mathrm{M}$. Coronal sections $(100 \mu \mathrm{m})$ were obtained and washed in cacodylate buffer, followed by a post-fixation using osmium tetroxide and uranyl acetate. Finally, the sections were embedded in Durcupan. Regions of the hippocampus were dissected under a stereoscopic microscope, mounted onto a blank resin slab, and trimmed using an ultramicrotome (Laica Ultracut UC-7). Imaging was performed using an NVision 40 FIB-SEM (Carl Zeiss) with an acceleration voltage of $1.5 \mathrm{kV}$, a current of $350 \mathrm{pA}$, and a dwell time of $10 \mu \mathrm{s} /$ pixel. Serial images were obtained using backscattered electrons and collected at a $6 \mathrm{~nm} /$ pixel magnification and $5 \mathrm{~nm}$ of milling depth between images.

\section{D reconstruction and rendering}

The serial micrographs were first registered using Multistackreg, a freely available plug-in for Fiji (47). Then, using those micrographs, we proceeded to the image segmentation and 3D model reconstructions by using TrackEM2 (a plug-in for Fiji) for manual segmentation, and iLastik, for a semi-automated segmentation. The extracted models were then imported to Blender software for visualization and rendering purposes (74).

\section{Extraction of tripartite synapse meshes}

For each synapse in contact with the $220 \mu \mathrm{m}^{3}$ astrocytic volume, a cube of edge length $1.5 \mu \mathrm{m}$ $\left(3.375 \mu^{3}\right)$ was created and centered at the center of mass of the PSD. All the elements of the mesh (astrocyte, astrocytic ER, spine and bouton) that were within the cubic volume were isolated using a boolean intersection operator available in Blender, forming what we refer to as a tripartite synapse mesh. The size of the cube was chosen to contain a single synapse (the neuropil is believed to contain around one synapse per micrometer cube) and large enough to contain the whole spine and bouton elements. This workflow resulted in the creation of 44 excitatory and 2 inhibitory synapse meshes. 


\section{Computational modeling}

\section{Modeled reactions and computational approach}

Astrocytic $\mathrm{Ca}^{2+}$ signals in PAPs were simulated using the reaction-diffusion voxel-based model of ER-dependent $\mathrm{Ca}^{2+}$ signaling from Denizot and colleagues ( (49) Table 2, Fig. 6-7). Briefly, the model describes $\mathrm{Ca}^{2+}$ fluxes in and out of the astrocytic cytosol. The opening of $\mathrm{IP}_{3} \mathrm{R}$ channels on the ER membrane triggers $\mathrm{Ca}^{2+}$ influx in the cytosol. $\mathrm{IP}_{3}$ can be synthesized by the $\mathrm{Ca}^{2+}$-dependent activity of Phospholipase C (PLC) $\delta$. $\mathrm{IP}_{3}$ removal from the cytosol is described by a decay rate. $\mathrm{IP}_{3} \mathrm{R}$ dynamics is derived from the De Young $\&$ Keizer's model (75). Each $\mathrm{IP}_{3} \mathrm{R}$ has 3 binding sites: one to $\mathrm{IP}_{3}$ and two to $\mathrm{Ca}^{2+}$ (activating and inhibiting). The channel can thus be in 8 different states. The open state is $\{110\}: \mathrm{IP}_{3}$ and $\mathrm{Ca}^{2+}$ bound to the activating sites and the $\mathrm{Ca}^{2+}$ inactivating site is unbound. In a subset of simulations, GCaMPs6s, genetically-encoded $\mathrm{Ca}^{2+}$ indicators (27), were added to the cytosol and variations of [CaGCaMP] concentration, mimicking experimental $\mathrm{Ca}^{2+}$ imaging, were measured. For further details on the kinetic scheme, parameter values and model assumptions, please refer to the original paper presenting the model (49). We slightly altered this model to better describe and control $\mathrm{IP}_{3} \mathrm{R}$-independent $\mathrm{Ca}^{2+}$ fluxes. To do so, $\mathrm{IP}_{3} \mathrm{R}$-independent $\mathrm{Ca}^{2+}$ influx was modeled as an influx through $\mathrm{Ca}^{2+}$ channels at the plasma membrane, $C h_{\mathrm{PM}}$. For simplicity, the amount of $C h_{\mathrm{PM}}$ channels equals the total number of $\mathrm{IP}_{3} \mathrm{R}$ channels, $N_{\mathrm{IP} 3 \mathrm{R}}$. $\mathrm{Ca}^{2+}$ influx rate at $C h_{\mathrm{PM}}$ channels, $\gamma_{\mathrm{ch}_{\mathrm{PM}}}$, is $15 \times 10^{-8} \mathrm{~s}^{-1}$. The reactions modeled here are illustrated in Fig. 4A.

The model was implemented using the STochastic Engine for Pathway Simulation (STEPS) python package (http://steps.sourceforge.net/) (76). This software uses a spatialized version of Gillespie's SSA algorithm (77) to perform exact stochastic simulations of reaction-diffusion systems. Simulations in STEPS allow the diffusion of molecules in 3D tetrahedral meshes and onto the surfaces of the mesh, such as the ER and plasma membrane. STEPS allows volume and surface reactions. Reactions can only occur between molecules within the same tetrahedron (volume reactions) or in adjacent triangle and tetrahedron (surface reactions). Boundary conditions were reflective. Simulation time was 100s. The states and amounts of all molecular species were measured at each time step (1 ms).

\section{Neuronal stimulation simulation}

Unless specified otherwise, glutamatergic transmission at the synapse was modeled and occurred at simulation time $\mathrm{t}=1 \mathrm{~s}$. To do so, $\mathrm{IP}_{3}$ molecules were injected in tetrahedra below the plasma membrane of the PAP, emulating $\mathrm{IP}_{3}$ synthesis resulting from the activation of metabotropic glutamatergic receptors at the membrane of the PAP. Supplemental movie 3 presents a visualization of a simulation at neuronal stimulation time, in the d2s6a9b1 PAP mesh. 


\section{$\mathrm{Ca}^{2+}$ channel clustering algorithm}

Surfaces correspond to triangular meshes. To simulate $\mathrm{IP}_{3} \mathrm{R}$ clustering, $N_{\mathrm{IP} 3 \mathrm{R}} / \eta \mathrm{IP}_{3} \mathrm{R}$ clusters were randomly positioned onto the membrane of the ER, where $N_{\mathrm{IP} 3 \mathrm{R}}$ is the total number of $\mathrm{IP}_{3} \mathrm{Rs}$ and $\eta$ is the number of channels per cluster. As $\eta$ is an integer, it must be a divider of $N_{\mathrm{IP} 3 \mathrm{R}}$. As $\mathrm{IP}_{3} \mathrm{R}$ density was kept constant across simulations, $3.5 e^{-3} / \mu m^{2}(49)$, the total number of $\mathrm{IP}_{3} \mathrm{Rs}, N_{\mathrm{IP} 3 \mathrm{R}}$, and $\mathrm{IP}_{3} \mathrm{R}$ cluster size $\eta$ varied depending on the mesh. Each $\mathrm{IP}_{3} \mathrm{R}$ cluster was located within a region of interest, as defined in STEPS, consisting in 4 triangles. Clusters could not overlap. In a subset of simulations, $\mathrm{IP}_{3} \mathrm{R}$ clusters were located at ER-PM contact sites. To do so, ER triangles were sorted depending on the distance between their center of mass and the closest PM triangle. Cluster center was then located at the ER triangle in which no cluster was already located characterized by the lowest ER-PM distance in the mesh. The cluster ROI consisted in this cluster center triangle and the neighboring triangles. Similarly, clusters could not overlap. The number of $\mathrm{IP}_{3} \mathrm{R}$ opening events at each cluster ROI was measured at each time step.

$\mathrm{IP}_{3} \mathrm{R}$ channels were co-clustered with $C h_{\mathrm{PM}} \mathrm{Ca}^{2+}$ channels at the plasma membrane (cocl=1), unless specified otherwise. If cocl $=0, C h_{\mathrm{PM}}$ channels were randomly distributed onto the plasma membrane. If cocl=1, $C h_{\mathrm{PM}}$ channels were co-clustered with $\mathrm{IP}_{3} \mathrm{Rs}$. To do so, $C h_{\mathrm{PM}}$ cluster center was defined as the triangle on the plasma membrane that was the closest to the $\mathrm{IP}_{3} \mathrm{R}$ cluster center triangle on the ER. The cluster ROI then consisted in this $C h_{\mathrm{PM}}$ cluster center and the neighboring triangles. Similarly to $\mathrm{IP}_{3} \mathrm{R}$ cluster ROIs, $C h_{\mathrm{PM}}$ clusters could not overlap. For simplicity, $C h_{\mathrm{PM}}$ cluster size was identical to $\mathrm{IP}_{3} \mathrm{R}$ cluster size: $\eta$.

\section{Simulation code}

Simulations were performed using the model of $\mathrm{Ca}^{2+}$ signals in fine processes from Denizot and collaborators (49), available at http://modeldb.yale.edu/247694.

\section{$\mathrm{Ca}^{2+}$ peak detection and characterization}

$\mathrm{Ca}^{2+}$ peaks were considered initiated and terminated when $\mathrm{Ca}^{2+}$ concentration increased above and decreased below peak threshold, respectively. Peak threshold was $[\mathrm{Ca}]_{\mathrm{b}}+n \sigma_{\mathrm{Ca}}$, where $[\mathrm{Ca}]_{\mathrm{b}}$ is the basal $\mathrm{Ca}^{2+}$ concentration and $\sigma_{\mathrm{Ca}}$ is the standard deviation of the $\left[\mathrm{Ca}^{2+}\right]$ histogram in the absence of neuronal stimulation. $\mathrm{n}$ varied depending on signal/noise ratio of the simulation of interest, notably when measuring Ca-GCaMP signals, noisier than free $\mathrm{Ca}^{2+}$ signals (see (e.g.) Fig 4E). $\mathrm{Ca}^{2+}$ peak frequency, duration and amplitude were measured in each simulation. $\mathrm{Ca}^{2+}$ peak duration corresponds to the time between peak initiation and termination, $\mathrm{Ca}^{2+}$ peak amplitude corresponds to the maximum number of $\mathrm{Ca}^{2+}$ ions in the cytosol measured within peak duration time and $\mathrm{Ca}^{2+}$ peak frequency corresponds to the amount of peaks detected during simulation time. The number of $\mathrm{IP}_{3} \mathrm{R}$ peak opening events was recorded at each time step, in the whole cell as well as at each $\mathrm{IP}_{3} \mathrm{R}$ cluster ROI. 


\section{D mesh manipulation}

All 3D mesh manipulations were performed with open-access, open-source software. All PAP 3D meshes used in this study will be available online upon paper acceptance.

\section{D PAP mesh processing for reaction-diffusion simulations}

PAP meshes from tripartite synapse meshes were pre-processed using Blender software so that they could be used for reaction-diffusion simulations. The workflow is illustrated in Fig. 4C. Intersection between ER and PAP membranes was prevented by using a boolean intersection operator. ER was relocated a few nanometers away from the plasma membrane. PAP compartments that did not belong to the main PAP volume were deleted. Boolean difference operation between PAP and ER elements was performed. Non-manifold vertices were repaired. The resulting PAP mesh was exported in .stl format, which was then converted into a .msh 3D tetrahedral mesh using TetWild software (78). Lastly, the mesh was imported into Gmsh software to be converted into 2.2 ASCII format, format supported by the STEPS mesh importer.

\section{Automated 3D PAP mesh generation}

We have implemented a workflow to generate realistic 3D tetrahedral PAP meshes characterized by various ER locations and constant ER shape. The algorithm is written in python, can be imported in Blender and will be available online upon paper acceptance. The workflow is presented in Fig. 8. First, all elements of the mesh, i.e the PAP and the ER, are relocated so that their center of mass is centered at the origin. Then, the ER is split into smaller ER objects using a custom-made function. Briefly, $\mathrm{n}$ cubes of a given size are placed along the ER object. Intersection boolean operation is then performed between the ER and each cube, resulting in the creation of $\mathrm{n}$ ER objects. ER objects smaller than $30 \mathrm{~nm}^{3}$ are deleted. The remaining ER objects are rescaled so that the sum of their surface areas matches the area of the original ER element, measured with the Blender 3D Print add-on. The number and size of cubes can be altered depending on the size of the original ER and on the mesh characteristics desired. Using Blender's physics engine, a simulation with $n$ frames is generated, in which ER objects are subject to physical forces that alter their location between each frame. Inputs of the 'RunPhysics' function include parameters that affect how close objects can get, which can be altered to prevent membrane intersection. Note that successful scattering of the ER depends on the geometrical properties of each mesh so that adjusting the parameters of physics simulation might be necessary depending on the mesh used. Details are provided in comments of the code to allow the user to adjust the code to the mesh under study. Examples of frames generated by this workflow applied to d1s15a32b1 PAP mesh is presented in Supplemental movie 2. For each selected frame, the mesh pre-processing steps presented in Fig. 4C are performed automatically, resulting in the export of a stl triangular mesh. 3D meshing and format conversion can then be performed using TetWild and Gmsh software, as described above. The resulting meshes can be used to perform reaction-diffusion simulations. 


\section{Analysis of the geometrical properties of 3D meshes}

The volume and surface area of each synaptic element, i.e the PAP, astrocytic ER, spine and bouton, were measured using the Blender add-on Neuromorph (79). We implemented a python script that can be imported in Blender software that measures distances between mesh elements of interest. The distance between each vertex of the plasma membrane of the PAP and the center of mass of the neighboring PSD was computed in Blender and stored in a list. Similarly, ER-PSD distance was quantified by measuring the distance between each vertex of the ER membrane and the center of mass of the PSD. To characterize ER-PM distance, for each vertex on the PM, the closest ER vertex was detected and its distance to the PM vertex was stored in a list. PM-PSD, ER-PSD and ER-PM distance lists were exported to a text file for analysis and visualisation.

\section{Statistical analysis}

Data analysis and statistics were performed using open-access and open-source software: the SciPy and pandas python libraries. Data visualization was performed using Seaborn and Matplotlib python libraries. Sample size for each analysis, n, is described in the figure legend. Prior to statistical analysis, normality of data distribution was inferred using the Shapiro-Wilk test. Relationship between $\mathrm{Ca}^{2+}$ peak characteristics and parameter values was inferred using one-way ANOVA if values followed a Gaussian distribution, Kruskal-Wallis one-way ANOVA otherwise. Note that the effect of $\mathrm{IP}_{3} \mathrm{R}$ clustering was quantified by measuring the ratio between the $\mathrm{Ca}^{2+}$ peak characteristic of interest measured at a given $\mathrm{IP}_{3} \mathrm{R}$ cluster size, $\eta>1$ and its mean value for $\eta=1$. The linear relationship between two datasets was evaluated using Spearman's correlation coefficient. The test and p-value, $p$, associated with each analysis is described in the figure legend or in the main text.

\section{References}

1. A. Verkhratsky, M. Nedergaard, Physiology of Astroglia. Physiological Reviews 98, 239389 (2018).

2. A. Araque, V. Parpura, R. P. Sanzgiri, P. G. Haydon, Tripartite synapses: glia, the unacknowledged partner. Trends in Neurosciences 22, 208-215 (1999).

3. I. Savtchouk, A. Volterra, Gliotransmission: Beyond Black-and-White. Journal of Neuroscience 38, 14-25 (2018).

4. E. A. Bushong, M. E. Martone, Y. Z. Jones, M. H. Ellisman, Protoplasmic astrocytes in CA1 stratum radiatum occupy separate anatomical domains. The Journal of Neuroscience: The Official Journal of the Society for Neuroscience 22, 183-192 (2002). 
5. M. R. Witcher, S. A. Kirov, K. M. Harris, Plasticity of perisynaptic astroglia during synaptogenesis in the mature rat hippocampus. Glia 55, 13-23 (2007).

6. I. Lushnikova, G. Skibo, D. Muller, I. Nikonenko, Synaptic potentiation induces increased glial coverage of excitatory synapses in CA1 hippocampus. Hippocampus 19, 753-762 (2009).

7. M. K. Herde, K. Bohmbach, C. Domingos, N. Vana, J. A. Komorowska-Müller, S. Passlick, I. Schwarz, C. J. Jackson, D. Dietrich, M. K. Schwarz, C. Henneberger, Local Efficacy of Glutamate Uptake Decreases with Synapse Size. Cell Reports 32, 108182 (2020).

8. C. Henneberger, L. Bard, A. Panatier, J. P. Reynolds, O. Kopach, N. I. Medvedev, D. Minge, M. K. Herde, S. Anders, I. Kraev, J. P. Heller, S. Rama, K. Zheng, T. P. Jensen, I. SanchezRomero, C. J. Jackson, H. Janovjak, O. P. Ottersen, E. A. Nagelhus, S. H. R. Oliet, M. G. Stewart, U. V. Nägerl, D. A. Rusakov, LTP Induction Boosts Glutamate Spillover by Driving Withdrawal of Perisynaptic Astroglia. Neuron (2020).

9. A. Badia-Soteras, T. S. Heistek, M. Kater, A. Negrean, H. S. Mansvelder, B. Khakh, R. Min, A. Smit, M. Verheijen, Proximity of astrocyte leaflets to the synapse determines memory strength, Tech. rep., bioRxiv (2022). Section: New Results Type: article.

10. A. Reichenbach, A. Derouiche, F. Kirchhoff, Morphology and dynamics of perisynaptic glia. Brain Research Reviews 63, 11-25 (2010).

11. Y. Bernardinelli, J. Randall, E. Janett, I. Nikonenko, S. König, E. V. Jones, C. E. Flores, K. K. Murai, C. G. Bochet, A. Holtmaat, D. Muller, Activity-dependent structural plasticity of perisynaptic astrocytic domains promotes excitatory synapse stability. Current biology: CB 24, 1679-1688 (2014).

12. A. Verkhratsky, M. Nedergaard, Astroglial cradle in the life of the synapse. Philosophical Transactions of the Royal Society B: Biological Sciences 369 (2014).

13. A. Araque, G. Carmignoto, P. G. Haydon, S. H. R. Oliet, R. Robitaille, A. Volterra, Gliotransmitters travel in time and space. Neuron 81, 728-739 (2014).

14. N. Mazaré, M. Oudart, J. Moulard, G. Cheung, R. Tortuyaux, P. Mailly, D. Mazaud, A.-P. Bemelmans, A.-C. Boulay, C. Blugeon, L. Jourdren, S. Le Crom, N. Rouach, M. CohenSalmon, Local Translation in Perisynaptic Astrocytic Processes Is Specific and Changes after Fear Conditioning. Cell Reports 32, 108076 (2020).

15. J. B. Foster, F. Zhao, X. Wang, Z. Xu, K. Lin, C. C. Askwith, K. J. Hodgetts, C.-L. Glenn Lin, Pyridazine-derivatives enhance structural and functional plasticity of tripartite synapse via activation of local translation in astrocytic processes. Neuroscience (2018). 
16. L. E. Ostroff, M. K. Manzur, C. K. Cain, J. E. LeDoux, Synapses lacking astrocyte appear in the amygdala during consolidation of Pavlovian threat conditioning. The Journal of comparative neurology 522, 2152-2163 (2014).

17. A. Panatier, D. T. Theodosis, J.-P. Mothet, B. Touquet, L. Pollegioni, D. A. Poulain, S. H. R. Oliet, Glia-Derived d-Serine Controls NMDA Receptor Activity and Synaptic Memory. Cell 125, 775-784 (2006).

18. A. Perez-Alvarez, M. Navarrete, A. Covelo, E. D. Martin, A. Araque, Structural and functional plasticity of astrocyte processes and dendritic spine interactions. The Journal of Neuroscience: The Official Journal of the Society for Neuroscience 34, 12738-12744 (2014).

19. C. Genoud, C. Quairiaux, P. Steiner, H. Hirling, E. Welker, G. W. Knott, Plasticity of Astrocytic Coverage and Glutamate Transporter Expression in Adult Mouse Cortex. PLoS Biology 4, e343 (2006).

20. J. Wenzel, G. Lammert, U. Meyer, M. Krug, The influence of long-term potentiation on the spatial relationship between astrocyte processes and potentiated synapses in the dentate gyrus neuropil of rat brain. Brain Research 560, 122-131 (1991).

21. J. P. Heller, D. A. Rusakov, The Nanoworld of the Tripartite Synapse: Insights from SuperResolution Microscopy. Frontiers in Cellular Neuroscience 11 (2017).

22. M. Arizono, V. V. G. K. Inavalli, S. Bancelin, M. Fernández-Monreal, U. V. Nägerl, Super-resolution shadow imaging reveals local remodeling of astrocytic microstructures and brain extracellular space after osmotic challenge. Glia 69, 1605-1613 (2021). _eprint: https://onlinelibrary.wiley.com/doi/pdf/10.1002/glia.23995.

23. D. A. Rusakov, Disentangling calcium-driven astrocyte physiology. Nature Reviews Neuroscience 16, 226-233 (2015).

24. A. Aboufares El Alaoui, M. Jackson, M. Fabri, L. de Vivo, M. Bellesi, Characterization of Subcellular Organelles in Cortical Perisynaptic Astrocytes. Frontiers in Cellular Neuroscience 14 (2021). Publisher: Frontiers.

25. A. Verkhratsky, A. Semyanov, R. Zorec, Physiology of Astroglial Excitability. Function 1 (2020).

26. A. Semyanov, C. Henneberger, A. Agarwal, Making sense of astrocytic calcium signals — from acquisition to interpretation. Nature Reviews Neuroscience pp. 1-14 (2020). Publisher: Nature Publishing Group.

27. E. Shigetomi, S. Patel, B. S. Khakh, Probing the Complexities of Astrocyte Calcium Signaling. Trends in Cell Biology 26, 300-312 (2016). 
28. A. Lia, V. J. Henriques, M. Zonta, A. Chiavegato, G. Carmignoto, M. Gómez-Gonzalo, G. Losi, Calcium Signals in Astrocyte Microdomains, a Decade of Great Advances. Frontiers in Cellular Neuroscience 15 (2021). Publisher: Frontiers.

29. L. Georgiou, A. Echeverría, A. Georgiou, B. Kuhn, Ca+ activity maps of astrocytes tagged by axoastrocytic AAV transfer. Science Advances 8, eabe5371 (2022). Publisher: American Association for the Advancement of Science.

30. A. Agarwal, P.-H. Wu, E. G. Hughes, M. Fukaya, M. A. Tischfield, A. J. Langseth, D. Wirtz, D. E. Bergles, Transient Opening of the Mitochondrial Permeability Transition Pore Induces Microdomain Calcium Transients in Astrocyte Processes. Neuron 93, 587-605.e7 (2017).

31. E. Bindocci, I. Savtchouk, N. Liaudet, D. Becker, G. Carriero, A. Volterra, Threedimensional $\mathrm{Ca}^{2+}$ imaging advances understanding of astrocyte biology. Science 356, eaai8185 (2017).

32. A. Asada, S. Ujita, R. Nakayama, S. Oba, S. Ishii, N. Matsuki, Y. Ikegaya, Subtle modulation of ongoing calcium dynamics in astrocytic microdomains by sensory inputs. Physiological Reports 3 (2015).

33. E. Shigetomi, E. A. Bushong, M. D. Haustein, X. Tong, O. Jackson-Weaver, S. Kracun, J. Xu, M. V. Sofroniew, M. H. Ellisman, B. S. Khakh, Imaging calcium microdomains within entire astrocyte territories and endfeet with GCaMPs expressed using adenoassociated viruses. The Journal of General Physiology 141, 633-647 (2013).

34. M.-Y. Sun, P. Devaraju, A. X. Xie, I. Holman, E. Samones, T. R. Murphy, T. A. Fiacco, Astrocyte calcium microdomains are inhibited by Bafilomycin A1 and cannot be replicated by low-level Schaffer collateral stimulation in situ. Cell Calcium 55, 1-16 (2014).

35. J. L. Stobart, K. D. Ferrari, M. J. P. Barrett, C. Glück, M. J. Stobart, M. Zuend, B. Weber, Cortical Circuit Activity Evokes Rapid Astrocyte Calcium Signals on a Similar Timescale to Neurons. Neuron 98, 726-735.e4 (2018).

36. R. Srinivasan, B. S. Huang, S. Venugopal, A. D. Johnston, H. Chai, H. Zeng, P. Golshani, B. S. Khakh, $\mathrm{Ca}(2+)$ signaling in astrocytes from Ip3r2(-/-) mice in brain slices and during startle responses in vivo. Nature Neuroscience 18, 708-717 (2015).

37. M. W. Sherwood, M. Arizono, C. Hisatsune, H. Bannai, E. Ebisui, J. L. Sherwood, A. Panatier, S. H. R. Oliet, K. Mikoshiba, Astrocytic IP3Rs: Contribution to Ca2+ signalling and hippocampal LTP. Glia 65, 502-513 (2017).

38. A. Panatier, J. Vallée, M. Haber, K. K. Murai, J.-C. Lacaille, R. Robitaille, Astrocytes are endogenous regulators of basal transmission at central synapses. Cell 146, 785-798 (2011). 
39. M. A. Di Castro, J. Chuquet, N. Liaudet, K. Bhaukaurally, M. Santello, D. Bouvier, P. Tiret, A. Volterra, Local $\mathrm{Ca} 2+$ detection and modulation of synaptic release by astrocytes. Nature Neuroscience 14, 1276-1284 (2011).

40. B. L. Lind, A. R. Brazhe, S. B. Jessen, F. C. C. Tan, M. J. Lauritzen, Rapid stimulus-evoked astrocyte $\mathrm{Ca} 2+$ elevations and hemodynamic responses in mouse somatosensory cortex in vivo. Proceedings of the National Academy of Sciences p. 201310065 (2013).

41. A. Panatier, M. Arizono, U. V. Nägerl, Dissecting tripartite synapses with STED microscopy. Phil. Trans. R. Soc. B 369, 20130597 (2014).

42. M. Arizono, V. V. G. K. Inavalli, A. Panatier, T. Pfeiffer, J. Angibaud, F. Levet, M. J. T. T. Veer, J. Stobart, L. Bellocchio, K. Mikoshiba, G. Marsicano, B. Weber, S. H. R. Oliet, U. V. Nägerl, Structural basis of astrocytic Ca 2+ signals at tripartite synapses. Nature Communications 11, 1-15 (2020). Number: 1 Publisher: Nature Publishing Group.

43. M. W. Sherwood, M. Arizono, A. Panatier, K. Mikoshiba, S. H. R. Oliet, Astrocytic IP3Rs: Beyond IP3R2. Frontiers in Cellular Neuroscience 0 (2021). Publisher: Frontiers.

44. I. Patrushev, N. Gavrilov, V. Turlapov, A. Semyanov, Subcellular location of astrocytic calcium stores favors extrasynaptic neuron-astrocyte communication. Cell Calcium 54, 343-349 (2013).

45. C. M. Kiyoshi, S. Aten, E. P. Arzola, J. A. Patterson, A. T. Taylor, Y. Du, A. M. Guiher, M. Philip, E. G. Camacho, D. Mediratta, K. Collins, E. Benson, G. Kidd, D. Terman, M. Zhou, Ultrastructural view of astrocyte-astrocyte and astrocyte-synapse contacts within the hippocampus. bioRxiv p. 2020.10.28.358200 (2020). Publisher: Cold Spring Harbor Laboratory Section: New Results.

46. L. H. Bergersen, C. Morland, L. Ormel, J. E. Rinholm, M. Larsson, J. F. H. Wold, A. T. Roe, A. Stranna, M. Santello, D. Bouvier, O. P. Ottersen, A. Volterra, V. Gundersen, Immunogold Detection of L-glutamate and D-serine in Small Synaptic-Like Microvesicles in Adult Hippocampal Astrocytes. Cerebral Cortex 22, 1690-1697 (2012).

47. C. Calì, J. Baghabra, D. J. Boges, G. R. Holst, A. Kreshuk, F. A. Hamprecht, M. Srinivasan, H. Lehväslaiho, P. J. Magistretti, Three-dimensional immersive virtual reality for studying cellular compartments in 3D models from EM preparations of neural tissues. Journal of Comparative Neurology 524, 23-38 (2016).

48. N. Medvedev, V. Popov, C. Henneberger, I. Kraev, D. A. Rusakov, M. G. Stewart, Glia selectively approach synapses on thin dendritic spines. Phil. Trans. R. Soc. B 369, 20140047 (2014). 
49. A. Denizot, M. Arizono, U. V. Nägerl, H. Soula, H. Berry, Simulation of calcium signaling in fine astrocytic processes: Effect of spatial properties on spontaneous activity. PLOS Computational Biology 15, e1006795 (2019).

50. I. Hepburn, R. Cannon, E. De Schutter, Efficient calculation of the quasi-static electrical potential on a tetrahedral mesh and its implementation in STEPS. Frontiers in Computational Neuroscience 7 (2013).

51. A. Denizot, M. Arizono, U. V. Nägerl, H. Soula, H. Berry, Spontaneous astrocytic calcium traces from organotypic hippocampal slices (2019). Publisher: figshare Type: dataset.

52. S. Zeller, S. Rüdiger, H. Engel, J. Sneyd, G. Warnecke, I. Parker, M. Falcke, Modeling of the modulation by buffers of $\mathrm{Ca} 2+$ release through clusters of IP3 receptors. Biophysical Journal 97, 992-1002 (2009).

53. S. M. Wiltgen, I. F. Smith, I. Parker, Superresolution localization of single functional IP3R channels utilizing Ca2+ flux as a readout. Biophysical Journal 99, 437-446 (2010).

54. N. B. Thillaiappan, A. Chavda, S. Tovey, D. Prole, C. Taylor, Ca2+ signals initiate at immobile IP3 receptors adjacent to ER-plasma membrane junctions. Nature Communications 8 (2017).

55. Taufiq-Ur-Rahman, A. Skupin, M. Falcke, C. W. Taylor, Clustering of InsP3 receptors by InsP3 retunes their regulation by InsP3 and Ca2+. Nature 458, 655-659 (2009).

56. I. F. Smith, S. M. Wiltgen, J. Shuai, I. Parker, Ca2+ Puffs Originate from Preestablished Stable Clusters of Inositol Trisphosphate Receptors. Sci. Signal. 2, ra77-ra77 (2009).

57. S. Rüdiger, J. Shuai, Modeling of Stochastic Ca2+ Signals. Computational Glioscience, M. De Pittà, H. Berry, eds., Springer Series in Computational Neuroscience (Springer International Publishing, Cham, 2019), pp. 91-114.

58. L. Lencesova, A. O’Neill, W. G. Resneck, R. J. Bloch, M. P. Blaustein, Plasma membranecytoskeleton-endoplasmic reticulum complexes in neurons and astrocytes. The Journal of Biological Chemistry 279, 2885-2893 (2004).

59. A. Denizot, H. Berry, S. Venugopal, Computational Modeling of Intracellular Ca2+ Signals in Astrocytes. Encyclopedia of Computational Neuroscience p. Submitted (2019). Submitted.

60. M. M. Wu, J. Buchanan, R. M. Luik, R. S. Lewis, Ca2+ store depletion causes STIM1 to accumulate in ER regions closely associated with the plasma membrane. J Cell Biol 174, 803-813 (2006). 
61. H. Wu, P. Carvalho, G. K. Voeltz, Here, there, and everywhere: The importance of ER membrane contact sites. Science 361, eaan5835 (2018).

62. C. Escartin, et al., Reactive astrocyte nomenclature, definitions, and future directions. Nature Neuroscience pp. 1-14 (2021). Publisher: Nature Publishing Group.

63. J. R. Jones, L. Kong, M. G. Hanna, B. Hoffman, R. Krencik, R. Bradley, T. Hagemann, J. Choi, M. Doers, M. Dubovis, M. A. Sherafat, A. Bhattacharyya, C. Kendziorski, A. Audhya, A. Messing, S.-C. Zhang, Mutations in GFAP Disrupt the Distribution and Function of Organelles in Human Astrocytes. Cell Reports 25, 947-958.e4 (2018).

64. E. Shigetomi, K. Saito, F. Sano, S. Koizumi, Aberrant Calcium Signals in Reactive Astrocytes: A Key Process in Neurological Disorders. International Journal of Molecular Sciences 20, 996 (2019).

65. T. Manninen, R. Havela, M.-L. Linne, Computational Models for Calcium-Mediated Astrocyte Functions. Frontiers in Computational Neuroscience 12 (2018).

66. A. Denizot, H. Berry, S. Venugopal, Intracellular Calcium Signals in Astrocytes, Computational Modeling of. Encyclopedia of Computational Neuroscience, D. Jaeger, R. Jung, eds. (Springer, New York, NY, 2020), pp. 1-12.

67. K. Breslin, J. J. Wade, K. Wong-Lin, J. Harkin, B. Flanagan, H. V. Zalinge, S. Hall, M. Walker, A. Verkhratsky, L. McDaid, Potassium and sodium microdomains in thin astroglial processes: A computational model study. PLOS Computational Biology 14, e1006151 (2018).

68. A. Denizot, M. Arizono, V. Nägerl, H. Berry, E. D. Schutter, Astrocyte nanoscale morphology controls Ca2+ signals at tripartite synapses. bioRxiv p. 2021.02.24.432635 (2021). Publisher: Cold Spring Harbor Laboratory Section: New Results.

69. L. Héja, Z. Szabó, M. Péter, J. Kardos, Spontaneous Ca2+ Fluctuations Arise in Thin Astrocytic Processes With Real 3D Geometry. Frontiers in Cellular Neuroscience 15 (2021). Publisher: Frontiers.

70. N. Korogod, C. C. Petersen, G. W. Knott, Ultrastructural analysis of adult mouse neocortex comparing aldehyde perfusion with cryo fixation. eLife 4, e05793 (2015).

71. M. Arizono, S. Bancelin, P. Bethge, R. Chéreau, A. Idziak, V. V. G. K. Inavalli, T. Pfeiffer, J. Tønnesen, U. V. Nägerl, Nanoscale imaging of the functional anatomy of the brain. Neuroforum (2021). Publisher: De Gruyter Section: Neuroforum.

72. R. Fernández-Busnadiego, Y. Saheki, P. D. Camilli, Three-dimensional architecture of extended synaptotagmin-mediated endoplasmic reticulum-plasma membrane contact sites. Proceedings of the National Academy of Sciences 112, E2004-E2013 (2015). 
73. T. Cui-Wang, C. Hanus, T. Cui, T. Helton, J. Bourne, D. Watson, K. Harris, M. Ehlers, Local Zones of Endoplasmic Reticulum Complexity Confine Cargo in Neuronal Dendrites. Cell 148, 309-321 (2012).

74. C. Calì, K. Kare, M. Agus, M. F. V. Castillo, D. Boges, M. Hadwiger, P. Magistretti, A Method for 3D Reconstruction and Virtual Reality Analysis of Glial and Neuronal Cells. JoVE (Journal of Visualized Experiments) p. e59444 (2019).

75. G. W. De Young, J. Keizer, A single-pool inositol 1,4,5-trisphosphate-receptor-based model for agonist-stimulated oscillations in $\mathrm{Ca} 2+$ concentration. Proceedings of the National Academy of Sciences 89, 9895-9899 (1992).

76. I. Hepburn, W. Chen, S. Wils, E. De Schutter, STEPS: efficient simulation of stochastic reaction-diffusion models in realistic morphologies. BMC Systems Biology 6, 36 (2012).

77. D. T. Gillespie, Exact stochastic simulation of coupled chemical reactions. The Journal of Physical Chemistry 81, 2340-2361 (1977).

78. Y. Hu, Q. Zhou, X. Gao, A. Jacobson, D. Zorin, D. Panozzo, Tetrahedral meshing in the wild. ACM Transactions on Graphics 37, 60:1-60:14 (2018).

79. A. Jorstad, B. Nigro, C. Cali, M. Wawrzyniak, P. Fua, G. Knott, NeuroMorph: A Toolset for the Morphometric Analysis and Visualization of 3D Models Derived from Electron Microscopy Image Stacks. Neuroinformatics 13, 83-92 (2015).

\section{Acknowledgements}

This work used the computing resources of the Scientific Computing and Data Analysis section from the Okinawa Institute of Science and Technology. We thank Iain Hepburn and Weiliang Chen of the Computational Neuroscience Unit, OIST, Okinawa, Japan for discussion and advice on STEPS software. We thank Pierre Magistretti (KAUST, Thuwal) for the financial support to MFVC and to CC, and Graham Knott for kindly sharing the original EM dataset.

Funding: The work from AD was supported by a JSPS (Japan Society for the Promotion of Science) Standard Postdoctoral Fellowship for Research in Japan (21F21733).

Author Contributions AD, CC and EDS conceived the research, provided resources and supervised the work. AD and EDS designed the analysis. MFVC and CC curated the electron microscopy data. PP wrote the Blender code for automatic 3D mesh handling. AD did the computational modeling work. AD performed data analysis and visualisation of experimental and computational data. AD wrote the first draft of the manuscript. All authors read and reviewed the manuscript. 
814 Competing Interests The authors declare that they have no competing financial interests.

816 Data and materials availability: The 3D meshes, geometrical data and code produced in this 817 study will be available online upon paper acceptance. 